\title{
ARTICLE OPEN \\ Dosage effect of multiple genes accounts for multisystem disorder of myotonic dystrophy type 1
}

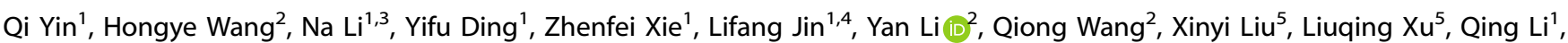
Yongjian Ma ${ }^{1}$, Yanbo Cheng ${ }^{1}$, Kai Wang ${ }^{1}$, Cuiqing Zhong ${ }^{1}$, Qian $\mathrm{Yu}^{6}$, Wei Tang ${ }^{6}$, Wanjin $\mathrm{Chen}^{5}$, Wenjun Yang ${ }^{1}$, Fan $\mathrm{Zhang}^{7}$, Chen Ding ${ }^{7}$, Lan $\mathrm{Bao}^{2}$, Bin $\mathrm{Zhou}^{2}$, Ping $\mathrm{Hu}^{2,8}$ and Jinsong $\mathrm{Li}$ (D) $^{1,9}$

Multisystem manifestations in myotonic dystrophy type 1 (DM1) may be due to dosage reduction in multiple genes induced by aberrant expansion of CTG repeats in DMPK, including DMPK, its neighboring genes (SIX5 or DMWD) and downstream MBNL1. However, direct evidence is lacking. Here, we develop a new strategy to generate mice carrying multigene heterozygous mutations to mimic dosage reduction in one step by injection of haploid embryonic stem cells with mutant Dmpk, Six 5 and Mbn/1 into oocytes. The triple heterozygous mutant mice exhibit adult-onset DM1 phenotypes. With the additional mutation in Dmwd, the quadruple heterozygous mutant mice recapitulate many major manifestations in congenital DM1. Moreover, muscle stem cells in both models display reduced stemness, providing a unique model for screening small molecules for treatment of DM1. Our results suggest that the complex symptoms of DM1 result from the reduced dosage of multiple genes.

Cell Research (2020) 30:133-145; https://doi.org/10.1038/s41422-019-0264-2

\section{INTRODUCTION}

Myotonic dystrophy type 1 (DM1) is a complex disease with variable pathological phenotypes, disease severities, and onset ages. ${ }^{1-3}$ The major symptoms include myotonia, muscle wasting, muscle weakness, cardiac conduction defects, cataracts and insulin resistance. ${ }^{3}$ DM1 is a genetic disease caused by the expansion of a CTG repeat in the $3^{\prime}$-untranslated region of the dystrophia myotonica protein kinase (DMPK) gene. ${ }^{4-6}$ Usually, with an increasing number of the repeats, respiratory failure and mental retardation can be observed in the most severe form of the disease (congenital DM1, CDM). ${ }^{7-10}$ A large number of studies have shown that the triple repeat expansion not only reduces the protein or mRNA levels of DMPK (haploinsufficiency model), ${ }^{11-13}$ but also alters the adjacent chromatin structure and reduces the expression of the neighboring genes, ${ }^{14-17}$ including downstream SIX5 $5^{18-22}$ and upstream DMWD ${ }^{23}$ in DM1 cells or patients (local chromatin structure change model), albeit with controversial observations. ${ }^{24-26}$ Moreover, numerous studies have also shown that nuclear-accumulated RNA containing the expanded CUG repeats aberrantly recruits splicing regulators, such as MBNL1, and forms the ribonuclear aggregates (foci) in nucleus (RNA toxicity model), leading to misregulation of alternative splicing. ${ }^{27-32}$ Mouse models carrying one mutation in
Dmpk, Six5 or Mbnl1 $1^{33-37}$ could only partially recapitulate the multisystem manifestations of DM1 patients. ${ }^{3}$ Moreover, the defects could only be observed in homozygous mutant mice (the affected proteins were completely absent), while the affected proteins were indeed present with a decreased level in human patients. Another set of DM1 mouse models are generated by overexpression of the CTG repeats; ${ }^{38}$ however, they could not fully recapitulate human phenotypes either. ${ }^{38}$ As an example, though the transgenic mouse model carrying human skeletal actin (HSA) gene and expressing 250 untranslated CUG repeats in the muscle $\left(H S A^{\mathrm{LR}}\right)$ can recapitulate multiple muscle phenotypes associated with DM1, it does not exhibit other common DM1related symptoms, such as muscle wasting and cataract. ${ }^{39}$ Meanwhile, this model cannot mimic CDM symptoms. ${ }^{39}$ The potential reason might be that the random insertion of transgenes in chromatin does not affect the expression of Dmwd-Dmpk-Six5 locus, which may be also involved in the development of complex symptoms of DM1. ${ }^{39}$ Moreover, muscle-specific overexpression of the repeats cannot mimic the state in human patients who carry the expanded repeats in whole body. Interestingly, a mouse model with an expanded human $(\mathrm{CTG})_{84}$ repeat inserted in the endogenous Dmpk gene displays no overt outward anomalies, ${ }^{40}$ suggesting that species-specific chromatin structure at Dmwd-

\footnotetext{
${ }^{1}$ State Key Laboratory of Cell Biology, Shanghai Key Laboratory of Molecular Andrology, CAS Center for Excellence in Molecular Cell Science, Shanghai Institute of Biochemistry and Cell Biology, Chinese Academy of Sciences, University of Chinese Academy of Sciences, Shanghai 200031, China; ${ }^{2}$ State Key Laboratory of Cell Biology, CAS Center for Excellence in Molecular Cell Science, Shanghai Institute of Biochemistry and Cell Biology, Chinese Academy of Sciences, University of Chinese Academy of Sciences, Shanghai 200031, China; ${ }^{3}$ Shanghai University of Medicine and Health Sciences affiliated Jiading District Central Hospital, Shanghai Key Laboratory of Molecular Imaging, School of Medical Technology, Shanghai University of Medicine and Health Sciences, Shanghai 201318, China; ${ }^{4}$ College of Life Science of Shaoxing University, Shaoxing, Zhejiang 312000, China; ${ }^{5}$ Department of Neurology and Institute of Neurology, First Affiliated Hospital, Fujian Medical University, Fuzhou, Fujian 350005, China; ${ }^{6}$ Animal Core Facility, CAS Center for Excellence in Molecular Cell Science, Shanghai Institute of Biochemistry and Cell Biology, Chinese Academy of Sciences, University of Chinese Academy of Sciences, Shanghai 200031, China; ${ }^{7}$ State Key Laboratory of Genetic Engineering, Human Phenome Institute, Institutes of Biomedical Sciences, School of Life Sciences, Zhongshan Hospital, Fudan University, Shanghai 200032, China; ${ }^{8}$ Institute for Stem Cell and Regeneration, Chinese Academy of Sciences, Beijing 100101, China and ${ }^{9}$ School of Life Science and Technology, Shanghai Tech University, Shanghai 201210, China

Correspondence: Ping Hu (hup@sibcb.ac.cn) or Jinsong Li (jsli@sibcb.ac.cn)
}

These authors contributed equally: Qi Yin, Hongye Wang, Na Li, Yifu Ding, Zhenfei Xie, Lifang Jin

Received: 10 April 2018 Accepted: 9 November 2019

Published online: 18 December 2019 
134

Dmpk-Six5 locus may result in different expression states of local genes, leading to distinct phenotypes in human and mouse. Taken together, mouse DM1 models that better recapitulate varieties of human symptoms are required to fully understand the underlying mechanism of DM1.

The multisystem symptoms of DM1 may be caused by the combination of different mechanisms, with down-regulation of multiple genes, including DMWD-DMPK-SIX5 and MBNL $1 .{ }^{41,42}$ However, the direct evidence to support this notion is missing due to challenges to generate mice carrying multiple gene mutations simultaneously. It is time- and labor-consuming to generate mice carrying triple or quadruple mutations using conventional methods. Recently, mouse androgenetic haploid embryonic stem cells (AG-haESCs) have been successfully developed as sperm replacement to efficiently produce semi-cloned (SC) animals by injection into oocytes (intracytoplasmic AG-haESC injection, ICAHCl). ${ }^{43-45}$ AG-haESCs enable efficient and stable onestep generation of mice with multiple heterozygous mutant genes by ICAHCl of haploid cells carrying these mutations, ${ }^{43}$ allowing production of sufficient numbers of SC offspring for analyses in one generation. Thus, the haploid ESC-mediated semi-cloning technology may provide an ideal tool to generate mouse models with multiple heterozygous mutant genes in one step to mimic the reduced expression of multiple genes in human complex diseases.

In this study, we tested our hypothesis by generating triple and quadruple heterozygous mutant mice in one step through injection of haploid ESCs carrying triple or quadruple mutant genes (Dmpk, Six5 and Mbn/1 or Dmpk, Six5, Mbnl1 and Dmwd) into oocytes and comparing these mutant mice with wild-type (WT) SC mice. Mice with triple mutations exhibited most of the major pathogenic phenotypes observed in adult-onset DM1 patients. Mice with quadruple mutations could mimic symptoms of patients from the most severe form of DM1, CDM. Interestingly, differentiation of muscle stem cells (MuSCs) is defective in both models due to stemness reduction, which recapitulates the defects in human DM1 patients. These MuSCs provide a novel system for screening drugs to treat muscle problems in DM1 in the future.

\section{RESULTS}

Generation of a novel DM1 model carrying mutations in Dmpk, Six5 and Mbnl1

We first examined the feasibility of semi-cloning technology to generate mouse models of DM1 carrying a single mutant gene by injection of haploid cells carrying a mutation in Dmpk, Six 5 or Mbnl1, the three well-studied DM1-related genes. A haploid cell line (termed $H_{1} 9^{\triangle D M R}-I G^{\Delta D M R}$-AGH or O48) that has been reported to efficiently support SC mouse generation ${ }^{43}$ was used in this study. We generated haploid ESC lines carrying mutant Dmpk, Six 5 or Mbnl1 (referred to as $\Delta$ Dmpk-O48, $\Delta$ Six5-O48 and $\Delta$ Mbnl1-O48, respectively) (Supplementary information, Figs. S1-3) and found that these cells efficiently supported the generation of live SC pups carrying heterozygous mutant Dmpk, Six 5 or Mbnl1 by ICAHCl (Supplementary information, Table S1). Mutant SC mice grew up normally to adulthood and showed similar growth curves to those of WT SC mice produced from 048 (Supplementary information, Figs. S1-3). Histological analysis of adult mice (4-6month old) showed no obvious phenotypic abnormality in the muscle of $\mathrm{Dmpk}^{+/-}$, Six $5^{+/-}$or $\mathrm{Mbn} / 1^{+/-}$SC mice (Supplementary information, Figs. S1-3).

We then set out to generate SC mice carrying triple mutations of Dmpk, Six5 and Mbnl1 in one step using ICAHCl. We disrupted Six 5 and Mbnl 1 in $\triangle$ Dmpk-O48-1 cells that have been analyzed as shown in Supplementary information, Fig. S1 and generated stable haploid cell lines carrying triple knockouts (termed $\triangle \mathrm{DSM}$ O48) (Fig. 1a, b). Whole-genome sequencing analysis showed no off-target effect in $\triangle \mathrm{DSM}$-048-1 cells (Supplementary information, Table S2). ICAHCl experiments showed that $\triangle \mathrm{DSM}-\mathrm{O} 48$ cells (from two cell lines, i.e., $\triangle D S M-048-1$ and $\triangle D S M-048-2$ ) could reproducibly produce live SC pups (termed DSM-TKO SC mice) after injection into oocytes (Fig. 1b; Supplementary information, Fig. S4a and Table S1). Over $90 \%$ of DSM-TKO SC pups grew up to adulthood and showed similar growth profiles to those of WT SC mice (Fig. 1c and Supplementary information, Table S1). We then examined muscle phenotypes commonly observed in DM1 patients, including myotonia, muscle weakness and muscle wasting, in DSM-TKO SC mice. Electromyography (EMG) test in skeletal muscle demonstrated myotonia in adult DSM-TKO SC mice (Fig. 1d). Mouse treadmill assay and grip strength test revealed that DSM-TKO SC mice displayed muscle weakness (Fig. 1e, f); and rotarod test indicated that DSM-TKO SC mice exhibited severe motor defects (Fig. 1g). Histological analysis of tibialis anterior (TA) muscles from DSM-TKO SC mice revealed several main histological hallmarks of muscles from DM1 patients, including increased number of nuclear clump and decreased fiber size (shown as the myofiber cross-sectional area (CSA), a sign of muscle wasting) (Fig. 1h, i). Meanwhile, consistent with the clinical observations, dystrophin (Dys) expression in DSM-TKO SC mice was normal (Supplementary information, Fig. S4b), while fiber type analysis indicated an increased ratio of type I (slow) myofiber and a decreased CSA of type I in DSM-TKO SC mice (Supplementary information, Fig. S4c). Abnormalities of diaphragm muscle and small intestine were also observed (Supplementary information, Fig. S4d, e), implying the potential breathing and digestive dysfunctions in DSM-TKO SC mice. We next analyzed the cardiac structure and function in adult DSM-TKO SC mice because heart abnormalities are common in DM1 patients. ${ }^{46}$ Echocardiography (ECG) did not show obvious structural and functional abnormalities in 4-month-old DSM-TKO SC mice (Supplementary information, Fig. S4f, g). However, 12-month-old DSM-TKO SC mice exhibited a significantly lower ejection fraction (Supplementary information, Fig. S4g), probably induced by increased myocardial fiber abnormalities in DSM-TKO SC mice (Supplementary information, Fig. S4h).

These data demonstrate that $\mathrm{Dmpk}^{+/-} ; \mathrm{Six}^{+/-} ; \mathrm{Mbnl1}^{+/-} \mathrm{SC}$ mice can be generated in one step using haploid cells carrying triple mutations. DSM-TKO SC mice mimic the phenotype of reduced dosage of three genes and display more severe pathological consequences compared to DM1 models carrying single homozygous mutation (Supplementary information, Table S3), providing a new model of DM1. However, DSM-TKO SC mice cannot mimic several phenotypes frequently observed in DM1 patients, such as cataracts and CDM symptoms, implying that other genes may be involved.

\section{$D M W D$ is involved in DM1}

It has been shown that expansion of the CTG repeats in DMPK produces allele-specific effects on transcription of the two adjacent genes, SIX5 (downstream of DMPK) and DMWD (upstream of $D M P K$ ) through changes of the local chromatin structure, leading to down-regulation of SIXS and DMWD in DM1 patients. ${ }^{14,18-21,23,47-51}$ The effects of SIX5 in DM1 have been well characterized using Six5-knockout mice; ${ }^{35,36,52}$ however, the role of DMWD in DM1 has not been analyzed yet. We set out to test the function of Dmwd by generating haploid cells carrying mutant Dmwd gene using CRISPR-Cas9 (Fig. 2a). ${ }^{53}$ Ten stable cell lines carrying mutant Dmwd gene (termed $\Delta$ Dmwd-O48) were obtained (Fig. 2b, c). By injecting these cells into oocytes, live SC pups could be efficiently produced (Fig. $2 \mathrm{~d}$ and Supplementary information, Table S1). Dmwd $d^{+-}$SC mice grew up to adulthood normally (Fig. 2e, f; Supplementary information, Table S1). Phenotype analysis showed that the myofiber CSA was dramatically reduced in adult $D m w d^{+/-}$mice (Fig. $2 \mathrm{~g}, \mathrm{~h}$ ), suggesting that DMWD is involved in pathological mechanism of DM1. However, other 
a

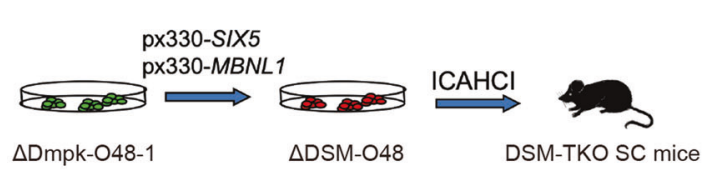

b

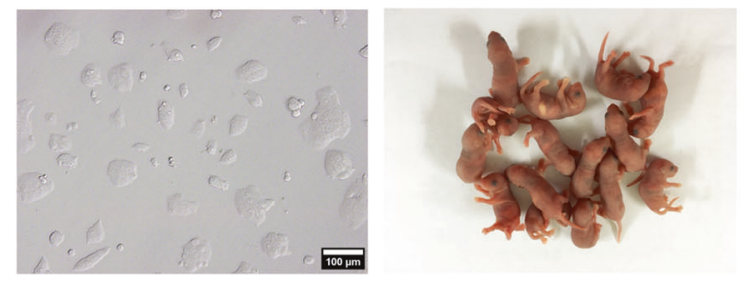

C

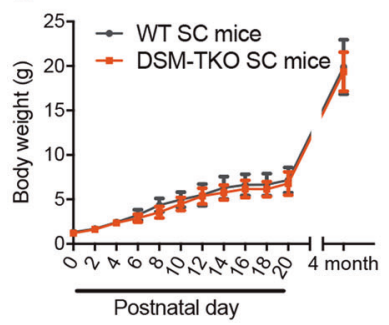

d

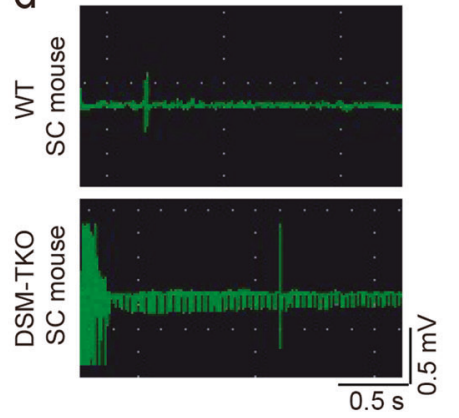

e

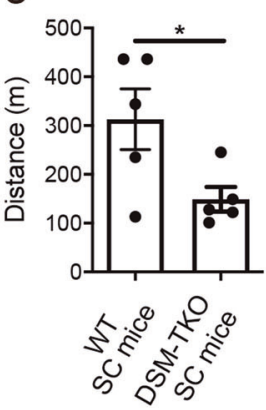

f

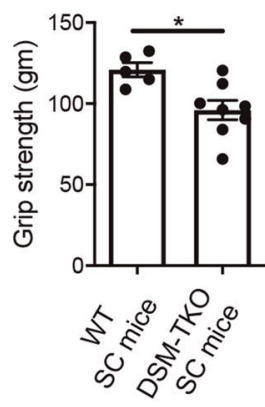

g

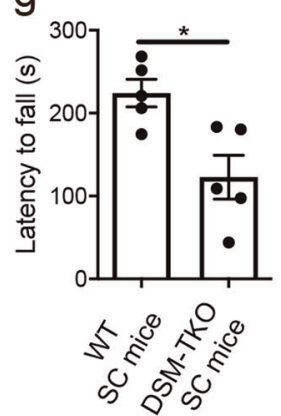

h

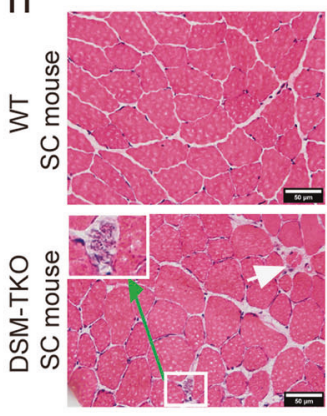

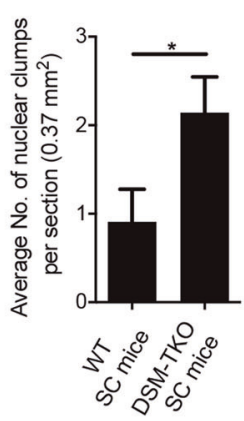

i

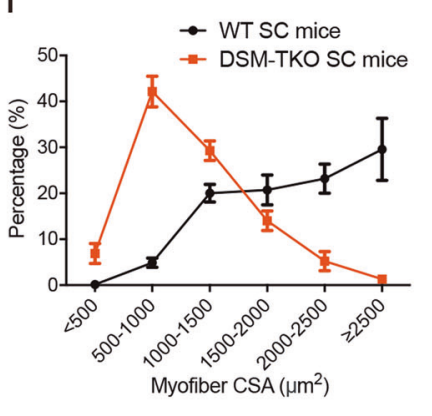

Fig. 1 SC mice carrying heterozygous mutations in Dmpk, Six5 and Mbnl1 exhibit typical DM1-associated muscle defects. a Diagram of DSMTKO SC mice generated by ICAHCl of $\triangle \mathrm{DSM}-\mathrm{O} 48$ cells carrying mutatnt Dmpk, Six 5 and Mbn/1. b Images of cultured $\triangle \mathrm{DSM}-\mathrm{O} 48-1$ haploid ESCs and newborn SC pups from $\Delta$ DSM-O48-1 cells. Scale bar, $100 \mu \mathrm{m}$. c Body weight analysis of DSM-TKO and WT SC mice $(n>5$ per group, means \pm SD). d EMG analysis of TKO SC mice (WT SC mice, $n=3$; DSM-TKO SC mice, $n=2$, 4-month old). e-g Muscle weakness of DSM-TKO SC mice was determined by treadmill test (e) ( $n=5$ per group, 4-month old), forelimb grip strength test (f) (WT SC mice, $n=5$; DSM-TKO SC mice, $n=$ 8 , 4-month old) and rotarod test $(\mathbf{g})\left(n=5\right.$ per group, 4-month old). Unpaired Student's $t$-test, ${ }^{*} P<0.05$. $\mathbf{h}$ H\&E staining of TA muscles from DSM-TKO and WT SC mice, DSM-TKO mice showing nuclear clump (green arrow) and atrophic fiber (white arrow) (WT SC mice, $n=11$; DSM-TKO SC mice, $n=7,4$-month old). Unpaired Student's $t$-test, ${ }^{*} P<0.05$. Scale bars, $50 \mu \mathrm{m}$. i TA myofiber CSA analysis $(n=3$ per group, 4-month old)

symptoms of DM1, such as cardiomyocyte defects, cataracts, and abnormalities of diaphragm muscle and small intestine (Fig. 2i), cannot be observed in Dmwd $d^{+/-}$mice, suggesting that DMWD is not sufficient to account for all the complex multisystem symptoms of DM1.

Mice carrying quadruple mutations show typical symptoms in CDM patients

Next, we tested whether compound loss of Dmpk, Six5, Mbnl1 and Dmwd could give rise to a mouse model recapitulating the most of the symptoms in DM1 patients and the more severe CDM manifestations. We further mutated Dmwd gene in $\triangle \mathrm{DSM}-\mathrm{O} 48-1$ cells and generated stable haploid cell lines carrying quadruple mutations (termed $\triangle \mathrm{DSMD}-\mathrm{O} 48$ ) (Fig. 3a). Off-target analysis indicated no unexpected mutation site in the tested cells ( $\triangle \mathrm{DSMD}-\mathrm{O} 48-2)$ (Supplementary information, Table S2). ICAHCI of three lines ( $\triangle D S M D-048-1,-2$ and -3 ) generated $\mathrm{Dmpk}^{+/-}$; $\mathrm{Six}^{+/-} ; \mathrm{Mbnl1}^{+/-}$; $\mathrm{Dmwd}^{+/-}$SC mice (DSMD-QKO SC mice) efficiently (Supplementary information, Fig. S5a, b and Table S1). In contrast to mice carrying single or triple mutations, $\sim 22 \%$ of newborn DSMD-QKO SC pups with normal body weights died in a couple of hours after birth (Fig. 3b). The autopsy indicated that the lung expansion failure might contribute to the neonatal death of DSMD-QKO SC pups (Supplementary information, Fig. S5c). Lung expansion failure can be caused by dysfunction of diaphragm muscle. We therefore dissected diaphragm from DSMD-QKO SC pups, which showed obvious pathogenic alterations, including reduced myofiber CSA and disorganized myofibers in diaphragm (Fig. 3c, d). Moreover, immunofluorescent staining analysis showed a significant decrease of the complexity of mature neuromuscular junctions (NMJs) in DSMD-QKO diaphragm muscles (Fig. 3e). Taken together, these results indicate that our DSMD-QKO SC mouse model recapitulates the postnatal respiratory insufficiency in CDM patients, which is the main cause of neonatal mortality of CDM patients. ${ }^{54}$ They also suggest that the postnatal respiratory insufficiency in CDM patients may be due to the defects of diaphragm muscle. ${ }^{55}$

We next characterized the growth profiles of DSMD-QKO SC mice. Interestingly, DSMD-QKO SC pups exhibited growthretarded phenotype (Supplementary information, Fig. S5d-f) and 


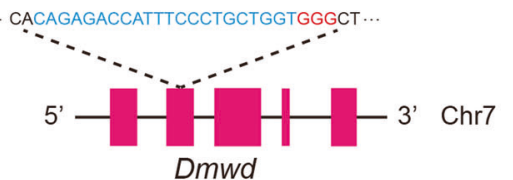

b

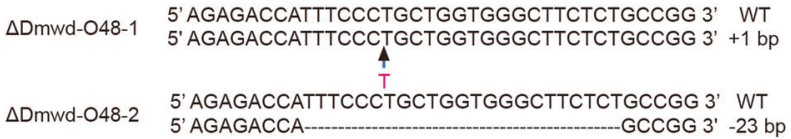

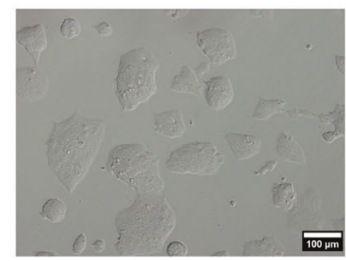

g
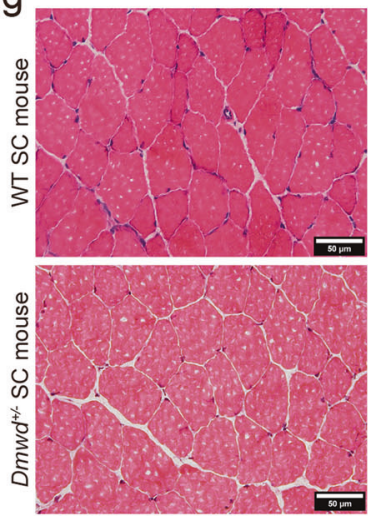

d

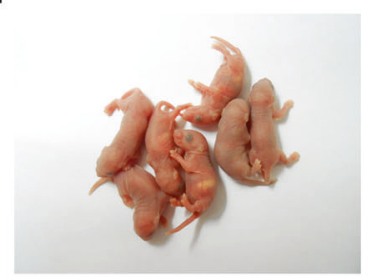

e

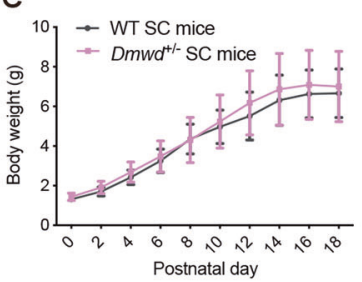

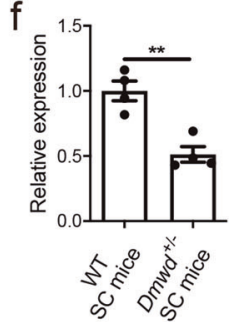

h

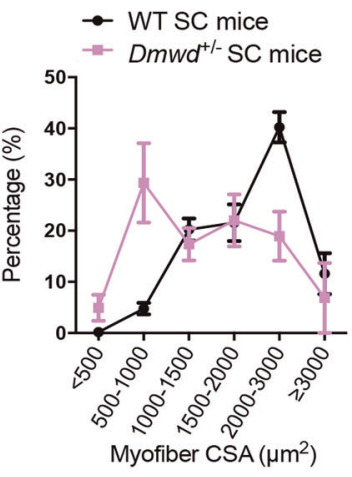

i
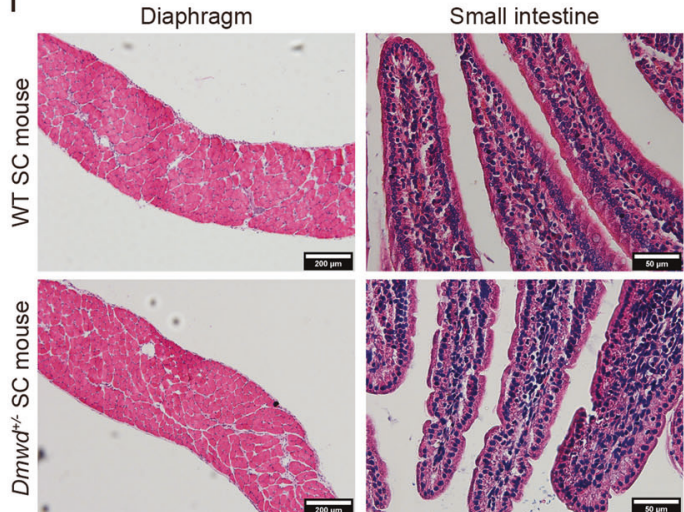

Fig. 2 Generation of $\mathrm{Dmwd}^{+/-}$SC mice through ICAHCl of haploid cells carrying mutant Dmwd. a Schematic of the sgRNA targeting Dmwd. b Sequences of the Dmwd gene in two cell lines ( $\triangle$ Dmwd-O48-1 and $\Delta$ Dmwd-O48-2) carrying CRISPR-Cas9-induced gene modifications. c Phase-contrast image of $\Delta$ Dmwd-O48-1 cell line. Scale bar, $100 \mu \mathrm{m}$. d Newborn SC pups generated from $\Delta$ Dmwd-O48-1 cells. e Average body weight of $\mathrm{Dmwd}^{+/-}$SC mice and WT SC mice $(n>8$ per group, means \pm SD). $\mathbf{f}$ Transcription analysis of Dmwd in TA muscles showed that the transcription level of Dmwd was significantly lower in Dmwd ${ }^{+/-}$SC mice compared with WT SC mice ( $n=4$ per group, 4-6-month old). Unpaired Student's $t$-test, ${ }^{*} P<0.01$. g Representative images of H\&E staining of TA muscles from DMWD ${ }^{+1-}$ SC mice and WT SC mice (4-6-month old). Scale bars, $50 \mu \mathrm{m}$. h CSA analysis of TA muscle showed muscle wasting in $D M W D^{+/}-\mathrm{SC}$ mice $(n=3$ per group, 4-6-month old). i Representative images of H\&E staining showed normal histological structure of diaphragm and small intestine in $D M W D^{+/-}$SC mice (4-6-month old). Scale bars, $200 \mu \mathrm{m}$ for diaphragm; $50 \mu \mathrm{m}$ for small intestine

$46 \%$ of them died within 3 weeks postnatally (Supplementary information, Table S1). We sacrificed DSMD-QKO SC pups on postnatal day 2 (P2) and observed residual milk in their stomachs, excluding the possibility that they died of feeding difficulties. P2 pups exhibited overt abnormal intestines (Supplementary information, Fig. S5g), consistent with the gastrointestinal abnormalities observed in CDM patients. ${ }^{54}$ Meanwhile, some of the DSMDQKO SC pups had abnormal talus bone development (Supplementary information, Fig. S5h), recapitulating the distinguishing feature of talipes in CDM patients. ${ }^{56}$ Neonatal hypotonia, another typical manifestation of CDM patients, ${ }^{54}$ was also observed in DSMD-QKO SC pups (Supplementary information, Fig. S5i), probably due to disorganized muscle fibers and less muscle mass (Fig. 3f). The percentage of myofibers containing centrally located nuclei increased in the mutant pups (Fig. $3 \mathrm{~g}$ ), which is a common symptom of DM1 reflecting constantly regenerating muscles with immature fibers. ${ }^{39,41,57}$ Cardiac problem is considered to be a common cause of sudden death in patients with DM1. ${ }^{46,58}$ We then performed ECG test and found that 2 of 7 mice (P17) exhibited ventricular premature beats (Supplementary information, Fig. S5j). Interestingly, these two pups died in a few days after ECG examination. Histological analysis of the heart showed the ventricular and atrial wall attenuation (Fig. 3h). Taken together, DSMD-QKO SC mice represent a novel model that can mimic developmental defects of CDM patients, suggesting that the reduced dosage of multiple genes is responsible for the CDM phenotypes and DMWD is involved in the development of DM1 symptoms.

Adult DSMD-QKO SC mice exhibit typical DM1 phenotypes DSMD-QKO SC mice, once survived at weaning, could grow up to adult (Supplementary information, Fig. S5d). Western blotting analysis confirmed the reduction of protein levels of DMPK, SIX5, MBNL1 and DMWD in the muscle of DSMD-QKO SC mice (Supplementary information, Fig. S6a, b). Mass spectrometry further confirmed protein reduction in adult QKO SC mice (Supplementary information, Fig. S6c). Interestingly, adult DSMDQKO SC mice exhibited adult DM1-associated muscle symptoms, including severe myotonia, muscle weakness and motor deficits (4-6-month old) (Fig. 4a, b; Supplementary information, Fig. S7a, b). Histological analysis indicated obvious structural abnormalities in limb muscles, including muscle wasting, and increased number of nuclear clump (Fig. 4c, d; Supplementary information, Fig. S7c, d). Similar histological abnormalities could also be observed in diaphragm muscle (Supplementary information, Fig. S7f). While QKO SC mice on P30 showed a high fraction of myofibers containing central nuclei (Fig. 4e and Supplementary information, Fig. S7e), this histological abnormality was milder with ages, 
a

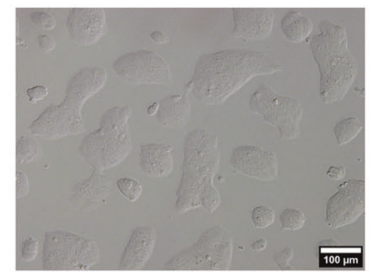

d
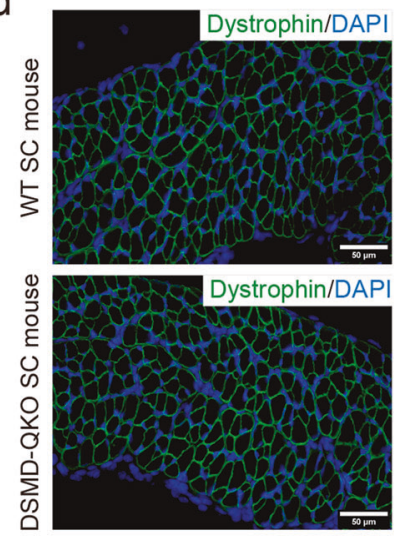

f

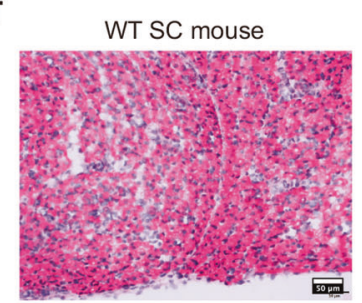

h

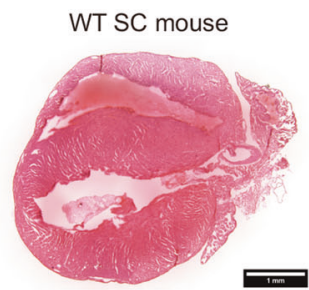

b
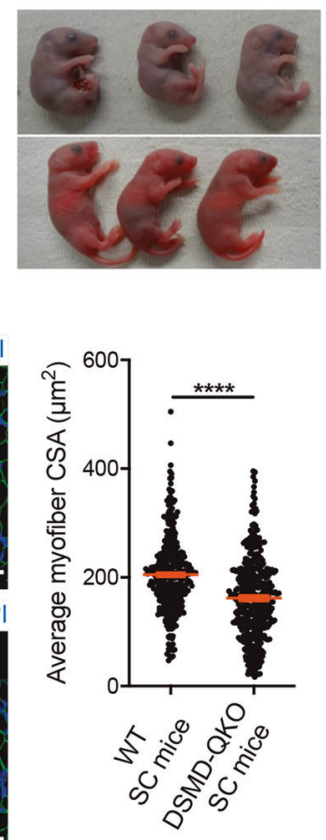

DSMD-QKO SC mouse

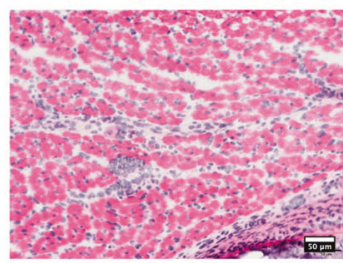

DSMD-QKO SC mouse

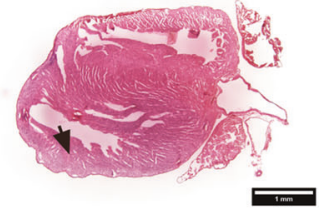

C

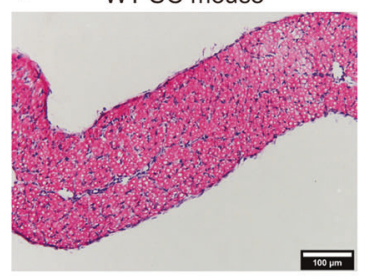

DSMD-QKO SC mouse
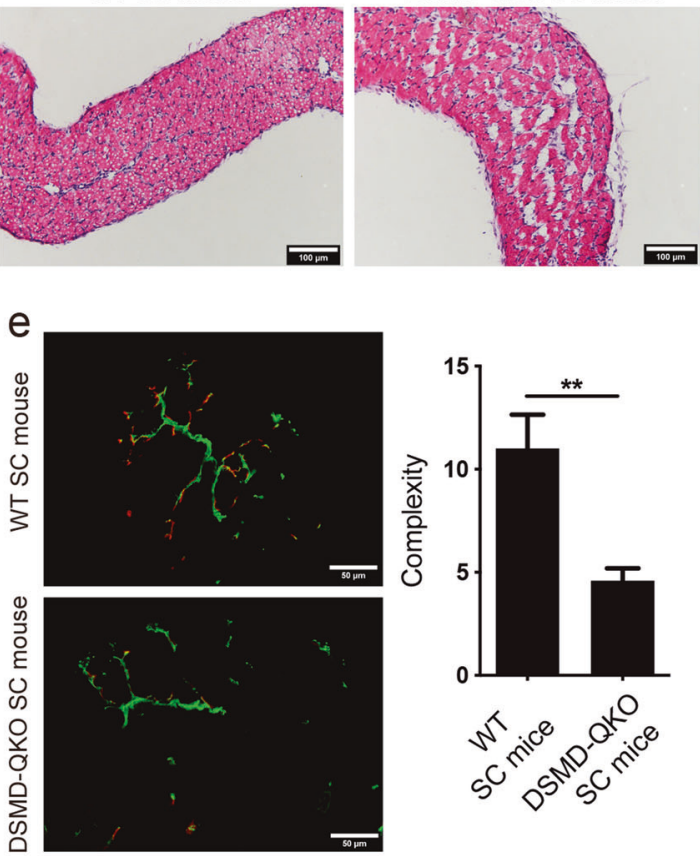

g

WT SC mouse

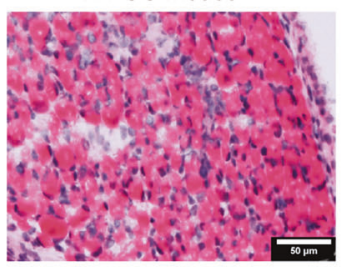

DSMD-QKO SC mouse
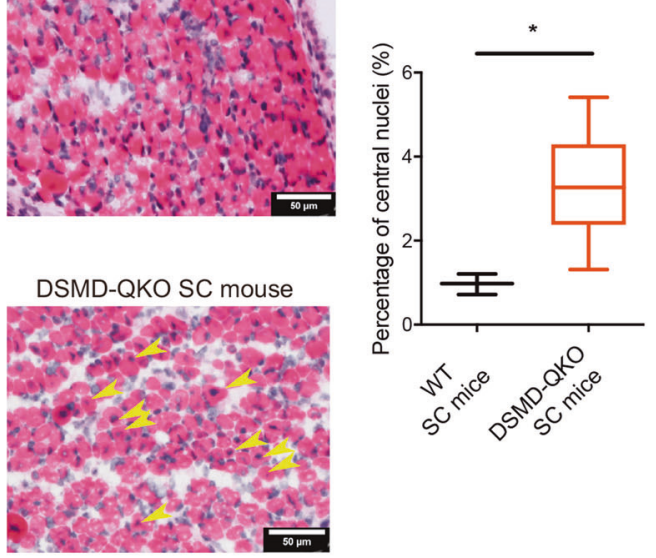

Fig. 3 SC mice carrying heterozygous mutations in Dmpk, Six5, Mbnl1 and Dmwd show CDM phenotypes. a Phase-contrast image of cultured $\triangle$ DSMD-048-1 haploid cells. Scale bar, $100 \mu \mathrm{m}$. b 23\% of newborn DSMD-QKO SC pups died during perinatal period (upper), while others survived (lower). c H\&E staining of diaphragm sections from DSMD-QKO and WT SC pups (P1). Scale bars, $100 \mu \mathrm{m}$. d Diaphragm CSA analysis of DSMD-QKO and WT SC mice (P1) $(n=3$ per group). Unpaired Student's $t$-test, $* * * * P<0.0001$. Scale bars, $50 \mu \mathrm{m}$. e Immunofluorescent staining of bungarotoxin (red) and neurofilament $\mathrm{H}$ (green), and complexity analysis of NMJs of DSMD-QKO and WT SC mice (P1) (WT SC pups, $n=2$; DSMD-QKO SC pups, $n=5$ ). Unpaired Student's $t$-test, ${ }^{* *} P<0.01$. Scale bars, $50 \mu \mathrm{m}$. $\mathbf{f} \mathrm{H} \& \mathrm{E}$ staining of TA muscle sections (P1) showing less myofiber in DSMD-QKO SC mice (2/5). Scale bars, $50 \mu \mathrm{m}$. g H\&E staining of TA muscle of DSMD-QKO and WT SC (P0) mice and the percentage of myofibers containing centrally located nuclei in TA muscle ( $n=3$ per group). Yellow arrows indicate the myofibers with central nuclei. Unpaired Student's $t$-test, ${ }^{*} P<0.05$. Scale bars, $50 \mu \mathrm{m}$. h H\&E staining of heart cryo-sections (P17) showing left ventricular posterior wall attenuation in DSMD-QKO SC mice (2/7, black arrow). Scale bars, $1 \mathrm{~mm}$

consistent with a phenotype in another DM1 mouse model generated by overexpression of CUGBP1. ${ }^{59}$ Meanwhile, fiber type analysis showed an increased percentage and a decreased CSA of type I myofibers in DSMD-QKO SC mice (Fig. 4f). Moreover, the complementary increase of type II (fast) myofiber CSA was also observed in QKO SC mice (Fig. 4f), representing another severe symptom in DM1 patients. ${ }^{60}$ In addition to muscle abnormalities, adult DSMD-QKO SC mice displayed other prominent DM1associated features, such as distinct dust-like cataracts (Fig. 4g) that is similar to the symptoms of DM1 patients, overt small intestine abnormalities ${ }^{54}$ and endocrine dysfunction displayed by big variation of endocrine hormone levels in different individuals (Supplementary information, Fig. S7g-i), mimicking the wide variations observed in patients. ${ }^{61}$ Although adult DSMD-QKO SC mice did not show major heart arrhythmias, they exhibited the overt functional defects and histological abnormalities in heart (Supplementary information, Fig. S7j-k).

Since mis-splicing is a characteristic feature of DM1 and the dosage of splicing factor Mbnl1 is also reduced in our models, we next analyzed splicing profiles of 11 DM1-related genes in P2, P10 and adult mice (4-month old). RT-qPCR analysis demonstrated the misregulation of $L d b 3$, Serca1, $m-T T N$, Tmem63b, Sorbs 1 and Spag9 


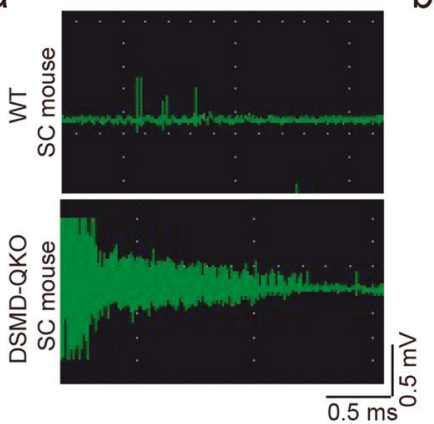

b

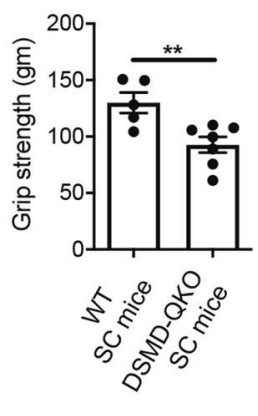

C

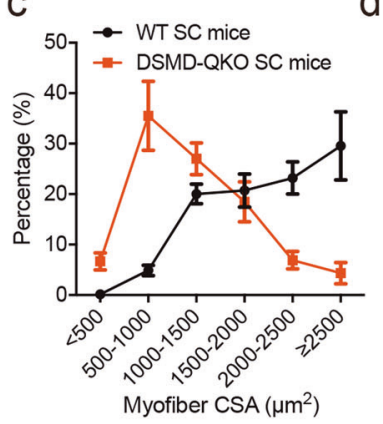

d

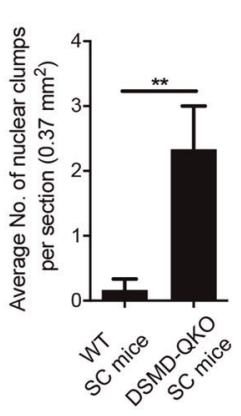

e

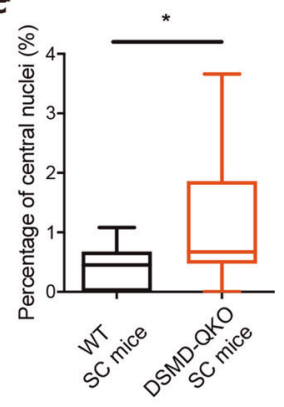

f

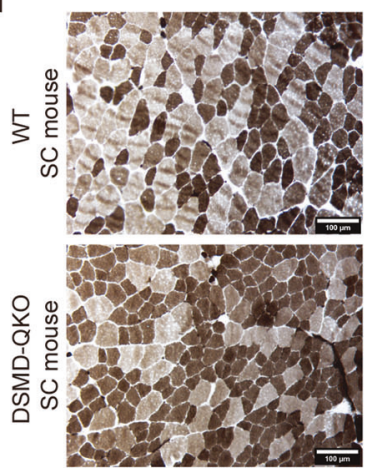

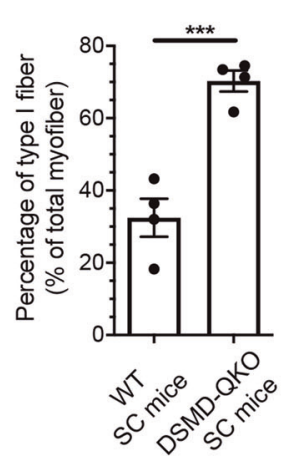
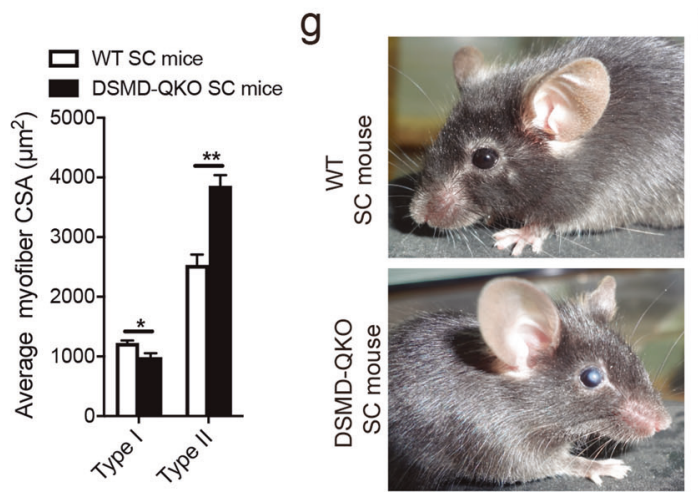

h

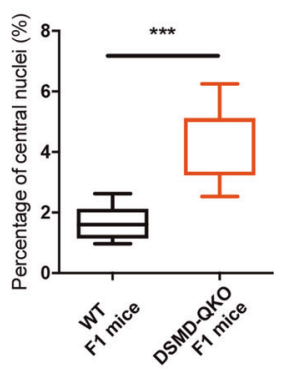

Fig. 4 DM1-related pathogenic phenotypes in adult SC mice carrying quadruple mutations. a EMG analysis indicates typical waxing-waning myotonia in QKO SC mice (WT SC mice, $n=3$; DSMD-QKO SC mice, $n=5,4-6$-month old). b Forelimb grip strength test (WT SC mice, $n=5$; DSMD-QKO SC mice, $n=7,4-6$-month old). Unpaired Student's $t$-test, ${ }^{* *} P<0.01$. c TA CSA analysis $(n=3$ per group, 4-6-month old). d Nuclear clump analysis of TA muscle (WT SC mice, $n=6$; DSMD-QKO SC mice, $n=3$, 4-6-month old). Unpaired Student's $t$-test, ${ }^{* *} P<0.01$. e The percentage of myofibers containing centrally located nuclei in TA muscle of QKO and WT SC mice (P30) ( $n=3$ per group). Unpaired Student's $t$-test, ${ }^{*} P<0.05$. f Representative images of ATPase staining and fiber type analysis ( $n=4$ per group, 4-6-month old). Unpaired Student's $t$-test, ${ }^{*} P<0.05,{ }^{*} P<0.01,{ }^{* * *} P<0.001$. Scale bars, $100 \mu \mathrm{m}$. g Dust-like opacities in eyes of DSMD-QKO SC mice (3/7, 4-6-month old). h The percentage of myofibers containing centrally located nuclei in TA muscles of QKO and WT F1 mice $(n=5$ per group, P0). Unpaired Student's $t$ test, ${ }^{* * *} P<0.001$

mRNA splicing, while the splicing efficiencies of Clcn1, Ryr1, Ryr2 and Tnnt3 mRNAs were normal (Supplementary information, Fig. S8; data not shown), reflecting the heterogeneity of missplicing in patients. ${ }^{62,63} \mathrm{~A}$ few mis-spliced genes observed in human patients appeared to be normal in our model, suggesting that other splicing factors such as MBNL2 ${ }^{64}$ and CUGBP1 ${ }^{59}$ might be involved in DM1.

Next, QKO F1 mice were obtained through regular mating with WT males (C57BL/6J background). Consistently, mRNA analysis showed the reduced expression of the four genes in both SC and F1 DSMD-QKO mice (Supplementary information, Fig. S9a). Interestingly, F1 QKO mice exhibited typical phenotypes shown in SC QKO mice, such as splicing abnormalities, TA and diaphragm muscle structure organization defects, and reduced muscle fiber size (Supplementary information, Fig. S9b-e). Furthermore, F1 QKO pups (P0) showed high percentage of the myofibers containing centrally located nuclei in TA muscles (Fig. 4h and Supplementary information, Fig. S9f). Taken together, DSMD-QKO SC mice can recapitulate most of the symptoms in DM1 and CDM (Supplementary information, Table S3), providing another new model for DM1 study. Furthermore, the defects can be passed to F1 of QKO SC mice, further suggesting that the DM1 symptoms caused by reduced dosage of multiple genes are stable.

MuSCs are defective in DSM-TKO and DSMD-QKO SC mice Previous studies have shown defects in MuSCs of DM1 patients, including aberrant stemness, ${ }^{65,66}$ abnormal proliferation ${ }^{65,67,68}$ and defective differentiation; ${ }^{65,69,70}$ we thus characterized the MuSCs in TKO and QKO SC mice. Immunofluorescent staining of
PAX7, the MuSC marker, indicated similar numbers of MuSCs in TKO, QKO (4-6-month old) and control SC mice at the same age (Fig. 5a). FACS analysis further confirmed that the ratio of MuSCs (CD34 ${ }^{+}:$integrin- $\left.\mathrm{a}_{7}^{+}: \mathrm{CD} 1^{-}: \mathrm{CD} 5^{-}: \mathrm{CD} 11 \mathrm{~b}^{-}: \mathrm{Sca}^{-}\right)^{71}$ were not affected in both DM1 models (Supplementary information, Fig. S10a). We then tested the in vitro proliferation ability of TKO and QKO MuSCs using the long-term expansion protocol established by Fu et al. ${ }^{72}$. The results showed that the proliferation abilities of both types of MuSCs were comparable to that from WT SC mice (Supplementary information, Fig. S10b, c).

We next analyzed the differentiation potentials of TKO and QKO MuSCs by culturing them in differentiation medium as reported previously. ${ }^{72}$ The results showed that the fusion index decreased in myofibers differentiated from both TKO and QKO MuSCs (Fig. 5b). In contrast, MuSCs from a well-established transgenic mouse model expressing long repeat of CUG $\left(H S A^{\mathrm{LR}}\right)$, which develops myotonia and myopathy, ${ }^{39}$ did not show differentiation defects in vitro (Supplementary information, Fig. S10c-e). To further confirm that the differentiation defect was cell autonomous, we performed MuSC transplantation experiments. We generated QKO SC mice carrying RFP transgene (termed RFPQKO SC mice) and control SC mice carrying EGFP transgene (EGFPWT SC mice) (Supplementary information, Fig. S11a, b) and then obtained MuSCs from the two strains, respectively. Consistent with the above results, RFP-QKO MuSCs showed normal proliferation ability and decreased in vitro differentiation potential (Supplementary information, Fig. S11c). We next transplanted RFP-QKO or EGFP-WT MuSCs to TA muscles of non-fluorescent WT recipients. The results showed that the CSA of $\mathrm{RFP}^{+}$myofibers (myofibers generated from the transplanted RFP-QKO MuSCs) was 
a

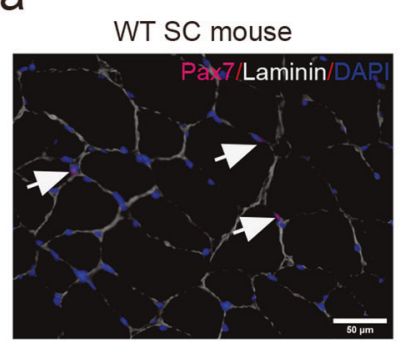

b
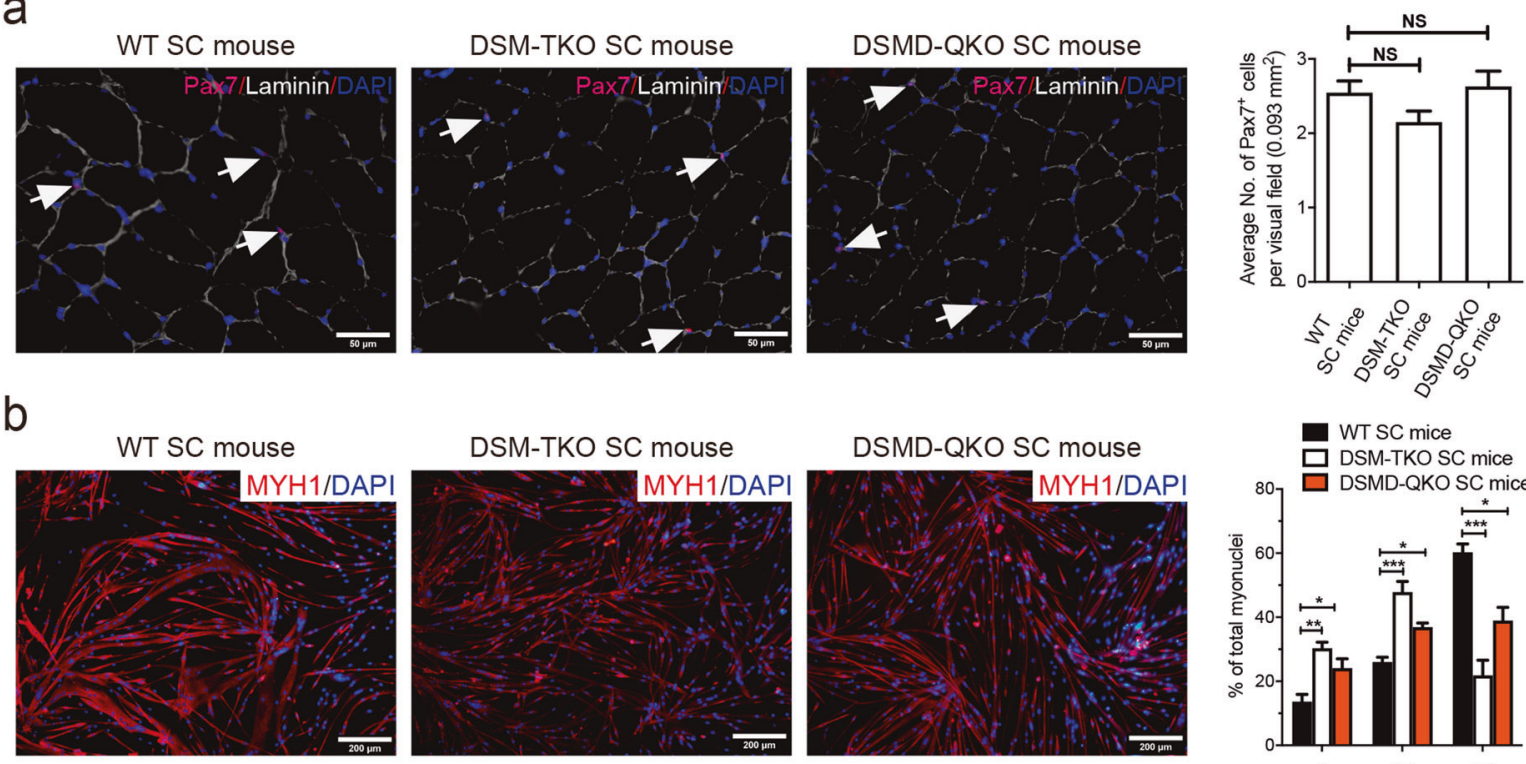

C
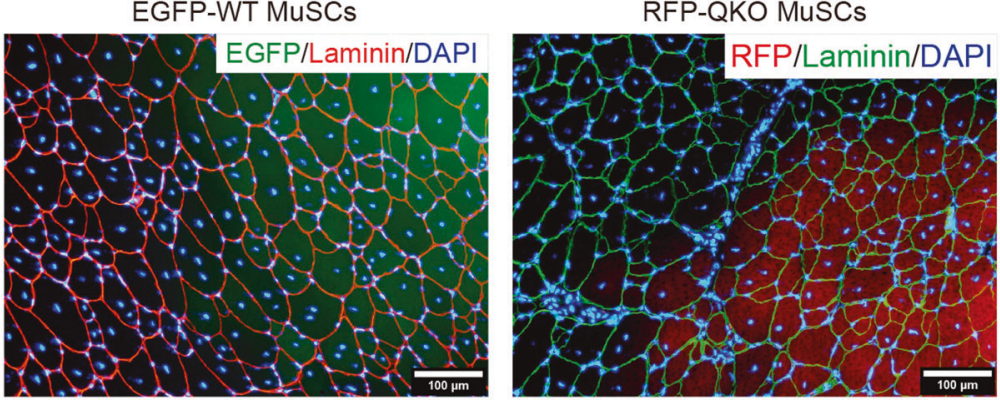

d
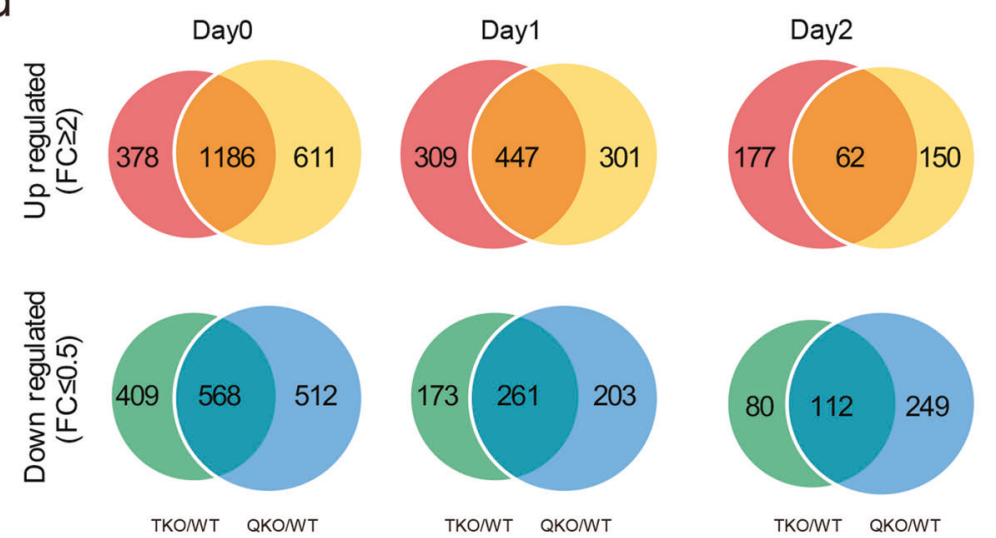

Dayo
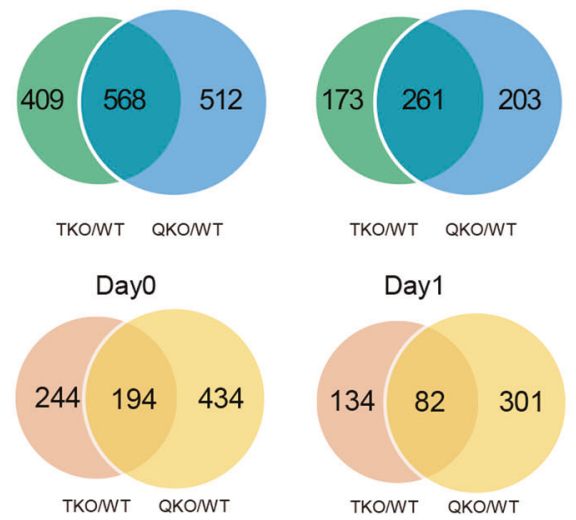

TKONWT QKONT

Day1

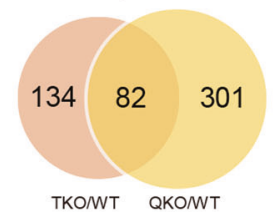

significantly smaller than that of the EGFP ${ }^{+}$myofibers generated from the transplanted EGFP-WT MuSCs (Fig. 5c). Since both types of MuSCs shared the same recipient microenvironment, we conclude that the differentiation defects of QKO MuSCs are cell autonomous. Together, these results demonstrate decreased
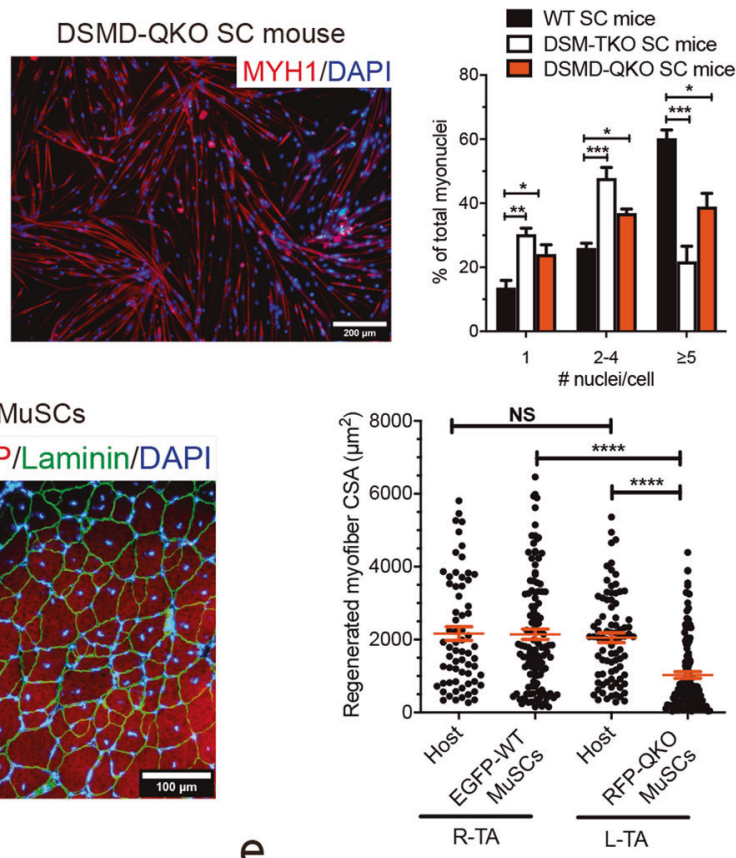

e

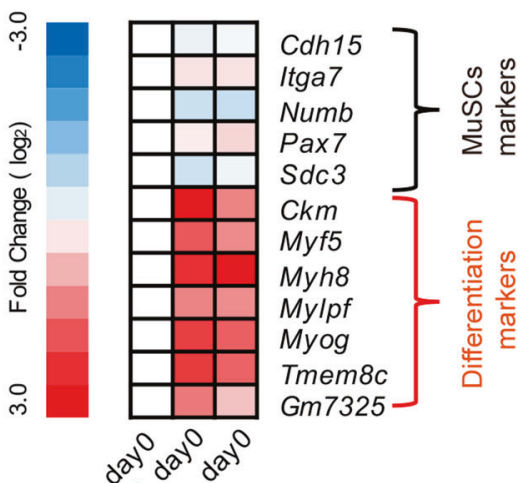

Day2

$112 \quad 53 \quad 339$

TKONT QKONT differentiation potential of MuSCs in TKO and QKO SC mice, mimicking another important symptom in DM1 patients.

We next attempted to reveal the underlying mechanisms by comparing the genome-wide gene expression profiles of MuSCs (Day 0) and differentiating cells (Day 1 and Day 2) between 
140

Fig. 5 Decreased differentiation potential of MuSCs from TKO or QKO DM1 mice. a Representative images of PAX7 immunostaining (pink) in TA muscle sections from adult TKO and QKO mice (4-6-month old, $n=3$ per group). White arrows indicate PAX7-positive cells. Scale bars, $50 \mu \mathrm{m}$. Unpaired Student's $t$-test. NS, no significant differences. b In vitro differentiation of MuSCs ( $n=3$ per group, 2-month old). One-way ANOVA, ${ }^{*} P<0.05,{ }^{*} P<0.01,{ }^{* * *} P<0.001$. Scale bars, $200 \mu \mathrm{m}$. c In vivo differentiation of EGFP-WT MuSCs and RFP-QKO MuSCs $(n=2$ per group, 2-month old). R-TA represents TA of right hind leg, while L-TA represents TA of left hind leg. One-way ANOVA, ${ }^{* * * * P<0.0001 . ~ N S, ~ n o ~}$ significant differences. Scale bar, $100 \mu \mathrm{m}$. d Venn diagrams show that the number of common DEGs between mutant and WT samples dramatically decreased following MuSC differentiation (from Day 0 to Day 2). e Heat map of stem cell markers and differentiation markers in

DM1 and WT MuSCs. $f$ Venn diagrams show that the number of common mis-splicing genes (skipped exon events) between mutant and WT samples dramatically decreased following MuSC differentiation (from Day 0 to Day 2)

mutant and normal SC mice (Supplementary information, Fig. S12a). Clustering analysis indicated that cells with the same differentiation stage rather than with the same genotype exhibited higher correlation (Supplementary information, Fig. S12b), suggesting that TKO and QKO mutations do not dramatically change the cell fate. Interestingly, the total number of differentially expressed genes (DEGs) between mutant and WT cells dramatically decreased after induction of differentiation (Fig. 5d), implying that the major differences existed at the stem cell stage. Enrichment analysis of DEGs on Day 0 showed that muscle differentiation-related genes (such as muscle development and contraction) were up-regulated in mutant MuSCs compared to WT cells (Fig. 5e; Supplementary information, Fig. S12c-e). In contrast, the expression levels of stem cell markers were similar (Fig. 5e; Supplementary information, Fig. S12d, e). Nevertheless, qRT-PCR analyses indicated that the expression levels of the muscle differentiation-related genes were comparable in differentiating cells from mutant and WT MuSCs (Supplementary information, Fig. S12f). We further analyzed the transcriptomewide splicing events from RNA-seq data and found that, consistent with the results in Fig. $5 \mathrm{~d}$, the mis-splicing genes mainly existed in MuSCs (Fig. $5 f$ and Supplementary information, Fig. S12g). These results demonstrate that MuSCs of TKO and QKO SC mice are at a more committed state. ${ }^{73}$ The premature expression of differentiation genes at stem cell stage suggests partial loss of stemness and may account for the decreased differentiation potential of mutant MuSCs in vitro and in vivo.

Having shown that our DM1 models exhibit the MuSC defects that may mimic DM1 symptoms, we finally investigated whether this property could be used for screening small molecules that can be potentially used to rescue the MuSC defects in DM1 patients in future due to availability of long-term in vitro maintenance and differentiation systems of mouse MuSCs. ${ }^{72}$ To this end, we developed a high-throughput platform for screening small molecules that may promote MuSC differentiation, in which, the 384-well plates were coated with collagen and planted with 3,500 TKO MuSCs, followed by differentiating treatment for two days in a differentiation medium (Fig. 6a). From a total of 10,000 compounds (Medical Research Council Technology), we identified 17 positive hits that could enhance the differentiation of TKO MuSCs. Further validation assays showed that T5381948 was the most potent small molecule to specifically promote the differentiation of TKO MuSCs in vitro (Fig. 6b, c). While in vivo validation is needed in future, these results suggest that our screening system based on TKO and QKO mouse models could be useful for potential development of drugs to treat DM1.

\section{DISCUSSION}

Mutations or expression changes occur in multiple genes in most of the diseases, especially in those multisystem syndromes. Generation of mouse models carrying multigene mutations to faithfully mimic the dosage reduction of these genes and recapitulate the complex symptoms is the key to understand and treat these syndromes (Fig. 6d). However, it is time consuming and labor intensive to do so using the conventional methods, leading to the lack of proper animal models for many syndromes. In this proof-of-concept study, we generated mouse models with multiple heterozygous mutations, which while do not occur in patients, can mimic the reduced expression of multiple genes in human DM1 that may be the underlying mechanisms of DM1 complex symptoms, in one step by ICAHCI of haploid ESCs carrying three or four mutant genes. Interestingly, mice carrying heterozygous mutant Dmpk, Six5 and Mbnl1 (DSM-TKO SC mice) to mimic dosage reduction exhibit typical symptoms of adult-onset DM1. With the additional haploid mutation in Dmwd, DSMD-QKO SC mice faithfully recapitulate the pathogenic phenotypes of the more severe CDM patients. These results suggest that dosage effects of multiple genes induced by different mechanisms, including haploidinsufficiency of DMPK, RNA toxicity and local chromatin changes, are the major pathogenic factors in DM1 and the severity of the disease depends on the number of genes affected. In patients, longer CTG repeats are associated with more severe symptoms and earlier disease onset; however, the underlying mechanism is unknown. Our results may provide potential explanation: besides abnormal RNA-induced sequestration of splicing factor MBNL1 as suggested by the RNA toxicity model, local chromatin structure changes induced by the aberrant expansion of CTG repeats in 3 -untranslated region of $D M P K$ gene first result in decreased expression of its downstream gene SIX5 (TKO SC mice); with an increasing number of repeats, the expression of its upstream gene DMWD is also affected (mimicked by QKO SC mice), leading to more severe and early onset DM1 (Supplementary information, Table S3). The longer the CTG repeat is, the more genes may be affected. The wide variety of human DM1 symptoms could be due to the variant contributions of different genes, which can weigh differently in individual patients, leading to their different phenotypes. To understand more about the mechanism of DM1 will help us group the patients more accurately for better treatment in future.

Given that F1 offspring from QKO SC mice exhibit typical DM1 symptoms (Fig. 4h and Supplementary information, S9), we tried to generate stable TKO or QKO mouse lines with or without H19/IG-DMR deletions through multiple backcross with C57BL/6J mice. Surprisingly, we found that heterozygous TKO or QKO mice without $\mathrm{H} 19$ and IG-DMR deletions (F3 and F4) did not exhibit typical DM1 symptoms. Interestingly, we unexpectedly observed upregulation of Dmpk, Six5, Mbnl1 and Dmwd in the animals (data not shown), which may account for the loss of phenotypes in these strains. A recent study has shown that genetic compensation in knockout zebrafish is activated by a premature termination codon-based knockout strategy, ${ }^{74}$ which has been used in our study and may induce the upregulation of WT allele of the targeted genes in F3 or F4 TKO/QKO mice during normal breeding. Meanwhile, we could not exclude the possibility that the potential dosage changes of genes in H19/lgf2 and Dlk1/Dio3 imprinted clusters induced by H19-DMR and IG-DMR deletions in DKO-AG-haESCs may partially account for the complex symptoms of the models. ${ }^{75}$ Moreover, while our models can recapitulate most symptoms of DM1 patients, they do not exhibit a few typical DM1 features, such as abnormal Clcn1 splicing, implying that other DM1-related genes including $M B N L 2^{76}$ and CUGBP $1^{59}$ might be involved in the disease. It will be interesting to test whether mouse models with modified Mbnl 2 and/or CUGBP1 can mimic these symptoms. 
a

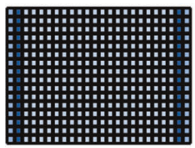

MuSCs planting ( $3.5 \mathrm{~K}$ )

(Control, DSM-TKO-1/2)

Collagen coating PBS washing Small molecules $(10 \mu \mathrm{M})$

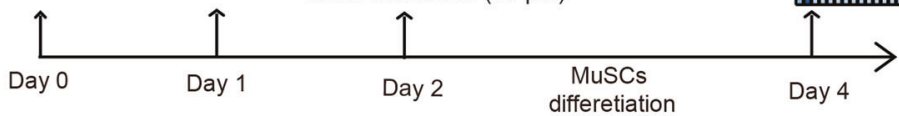

b
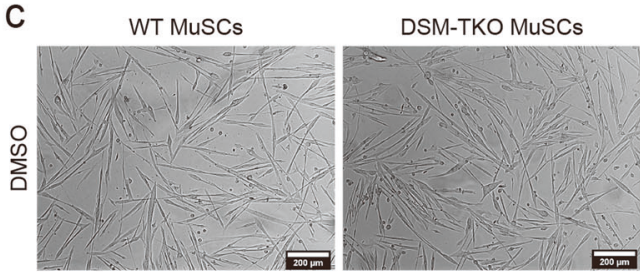

DSMD-QKO MuSCs<smiles>CCN(CC)c1ccc(-c2n[nH]c(=S)n2-c2ccccc2C)cc1</smiles>

Enamine: T5381948
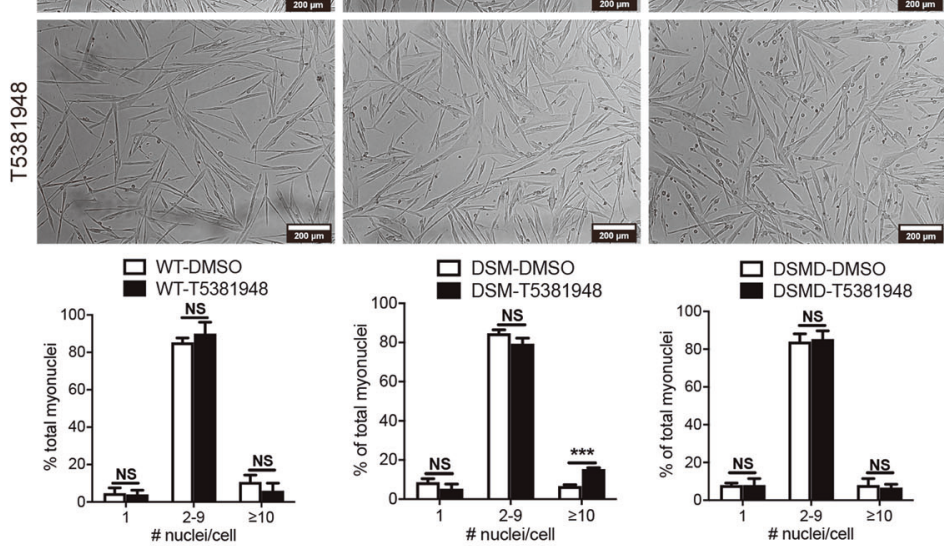

d

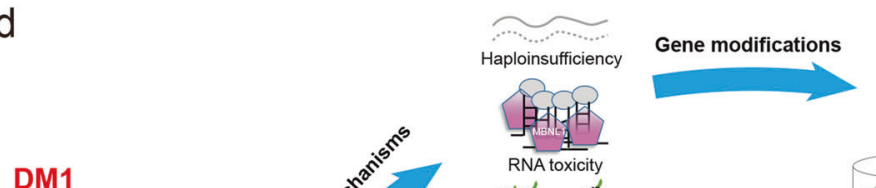

DM1

Patient
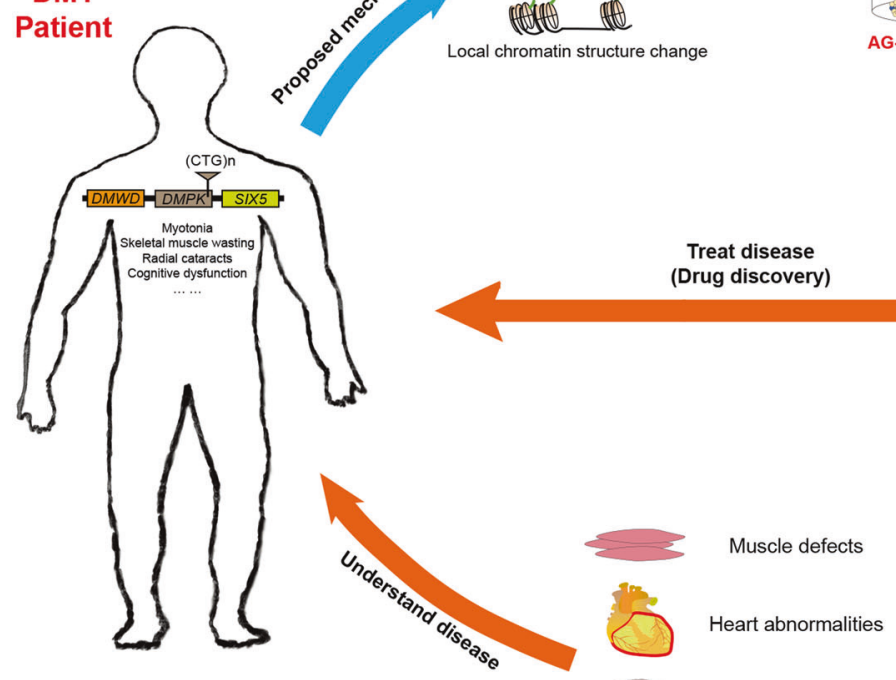
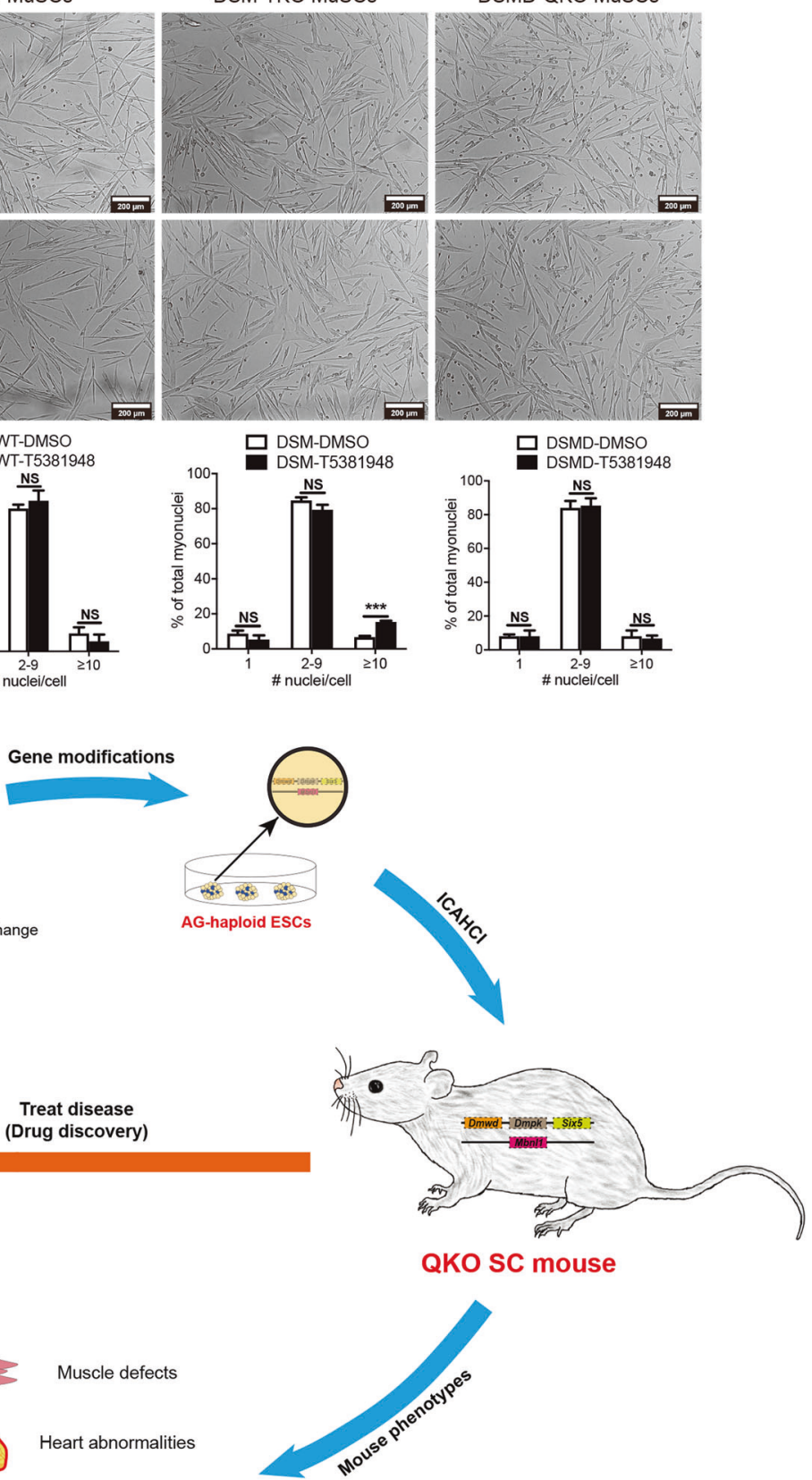

Endocrine disorders

Endocrine disorders
Cognitive dysfunction

$$
\text { ...... }
$$

Fig. 6 Large-scale screening and potential applications of DM1 mice. a Diagram of large-scale screening for small molecules that can promote differentiation of DM1 MuSCs. b Molecular structure of T5381948. c Images show that T5381948 could specifically improve the in vitro differentiation of DSM-TKO MuSCs. Scale bars, $200 \mu \mathrm{m}$. Unpaired Student's $t$-test; ${ }^{* * *} P<0.001$; NS, no significant differences. d Overview of the generation and applications of DM1 mouse model with quadruple heterozygous mutations 
Defects in MuSCs have been shown to be related to DM1 and CDM patients. ${ }^{65,67,77}$ However, this pathological phenotype has not been recapitulated in mice yet until in 2017 during preparation of our manuscript, Thomas et al. ${ }^{26}$ reported that conditional disruption of $\mathrm{Mbn} / 3$ in muscle resulted in defective MuSC differentiation. However, the expression of MBNL3 is not affected in DM1 patients, implying that the phenotype observed in their study is not closely related to the MuSC defects observed in DM1 patients. In contrast, our TKO and QKO models generated by mimicking dosage reduction of multiple DM1-related genes in mice, exhibited defective in vitro and in vivo differentiation phenotypes, and thus might truly reflect the state in patients. Meanwhile, this property enables a unique system to screen small molecules that enhance the differentiation of DM1 MuSCs in vitro; such molecules may also improve the MuSC differentiation in vivo, ameliorating the muscle defects induced by less stemness in patients, such as muscle weakness and wasting. Taken together, our study suggests that abnormal expansion of CTG repeats in $D M P K$ could disrupt the chromatin structure and affect the neighboring genes, which, combined with aberrantly expanded DMPK mRNA-mediated RNA toxicity mechanism, accounts for complex pathogenic phenotypes in DM1 patients.

Our method paves the road towards generation of a series of mouse models carrying multiple gene mutations in a short time to mimic different stages and severities of the complex syndromes. A potential application of the approach is to produce mice carrying multiple modifications in candidate loci that have been identified in high-throughput studies or genetic screenings to mimic clinical manifestations of multigenic diseases. ${ }^{78}$ In summary, we have established novel DM1 mouse models by one-step injection of haploid cells carrying three or four mutant genes into oocytes, providing suitable models to investigate the molecular mechanisms underlying complex manifestations and perform drug screening (Fig. 6d). Future analysis of the TKO and QKO mice will reveal more genes involved in the complex manifestations of DM1. Meanwhile, we hope that our haploid ESC-mediated semicloning technology will promote modeling of other complex diseases in mice.

\section{MATERIALS AND METHODS}

Experimental mice

All animal procedures were carried out in accordance with the guidelines of the Shanghai Institute of Biochemistry and Cell Biology (SIBCB). All mice were housed in specific pathogen-free facilities of SIBCB. Oocytes for micromanipulation were obtained from female mice of $\mathrm{B} 6 \mathrm{D} 2 \mathrm{~F} 1$ (C57BL/6J $\times \mathrm{DBA} / 2)$ background. $H S A^{L R}$ mice (FVB/n background) and $I L 2 r^{-1-}$ mice (C57BL/6J background) were purchased from the Jackson Laboratory.

Derivation of gene-modified haploid cell lines

CRISPR-Cas9-mediated gene manipulation was performed, as previously described. ${ }^{53,79}$ Briefly, oligos for different genes (Dmpk, Six5, Mbnl1 and Dmwd) were synthesized and ligated in px330mcherry plasmid. Constructed plasmids were then transfected into haploid cells (O48), which were cultured in the ESC medium plus $3 \mu \mathrm{M}$ CHIR99021 and $1 \mu \mathrm{M}$ PD0325901 (ES + 2i). The haploid cells were enriched through FACS and plated in one well of the 6-well plate at low density to obtain single-cell clone in $24 \mathrm{~h}$ after transfection. 6-7 days after plating, the single-cell clones were picked and separated into two parts, one for passaging and the other for sequencing to determine the genotype. For generation of EGFP-O48 and RFP- $\triangle D S M D-048$ haploid ESCs, the haploid ESCs were transfected with PiggBac plasmids (CAG-EGFP or CAG-RFP).

\section{$\mathrm{ICAHCl}$}

To produce SC embryos, the haploid ESCs arrested in M phase by culturing in medium containing $0.05 \mu \mathrm{g} / \mathrm{mL}$ demecolcine were trypsinized and resuspended in Hepes-CZB medium, then injected into oocytes using a Piezo-drill micromanipulator (Prime Technology Ltd), as described previously. ${ }^{80}$ The $\mathrm{SC}$ embryos were cultured in $\mathrm{KSOM}$ medium with amino acids at $37^{\circ} \mathrm{C}$ under $5 \% \mathrm{CO}_{2}$ in air. The 2-cell stage embryos were transferred into oviduct of pseudopregnant ICR females at 0.5 days post coitum with 15-20 embryos per side. Recipient mothers naturally delivered the SC pups.

\section{Immunostaining}

Dissected tissues from diaphragm, heart and TA muscle were put into the OCT (Leica) directly, and then frozen in liquid nitrogen for $15 \mathrm{~s}$. The frozen tissues could be stored in $-80^{\circ} \mathrm{C}$ refrigerators until sectioning. For whole heart longitudinal sections, the freshly dissected tissues were washed with PBS and fixed with $4 \%$ paraformaldehyde (PFA) at $4{ }^{\circ} \mathrm{C}$ for $30 \mathrm{~min}$. After being dehydrated with $30 \%$ sucrose solution, the samples were embedded in OCT. The protocol for Pax7 (DHSB, 1:200) staining was carried out according to the previously described protocols. ${ }^{72,81}$ Immunostaining for dystrophin was performed using anti-dystrophin (Abcam, ab15277) as primary antibody (1:400) and Alexa 488-conjunated anti-mouse antibody (Invitrogen) as secondary antibody. All images were acquired on Leica SP8 confocal microscope. For NMJ structural analysis, the frozen transverse sections of diaphragm were stained with a-bungarotoxin conjugated with Alexa Fluor 594 (Invitrogen, B13423) for acetylcholine receptors, and then incubated with chicken ployclonal antibody for neurofilament $\mathrm{H}$ (EnCor Biotechnology, CPCA-NF-H, 1:2000), followed by incubation with Alexa 488labeled secondary antibody.

Dissection and H\&E staining of mouse TA muscle

TA muscle was gently removed from the mouse hindlimb after peeling off the leg skin. After dissection, TA muscles were embedded with minimum of Tissue-Tec O.C.T compound and quickly frozen with liquid nitrogen. These tissues could be stored in $-80^{\circ} \mathrm{C}$ freezer before cryosectioning. Around $10 \mu \mathrm{m}$-thick sections were made and collected on positively charged microscope slides. Then the slides were fixed with methanol before being subjected to hematoxylin and eosin (H\&E) staining. After the slides were mounted with mounting media, the images were captured with Olympus BX51 microscope. The myofiber CSA and the percentage of myofibers with central nuclei in TA muscles were analyzed with ImageJ.

\section{Histology}

Frozen transverse sections $(10 \mu \mathrm{m})$ of TA and diaphragm muscles were stained with H\&E. Fiber CSAs were measured using ImageJ. ATPase staining was performed, as described previously. ${ }^{82}$ Briefly, transverse sections were incubated in $\mathrm{pH} 4.6$ solution for 5-15 min, followed by staining with ATP solution. After being washed with medium containing $1 \% \mathrm{CaCl}_{2}(\mathrm{w} / \mathrm{v}), 2 \% \mathrm{CoCl}_{2}(\mathrm{w} / \mathrm{v})$, $0.1 \mathrm{M}$ Sodium Barbital solution (1:20) and $1 \%$ ammonium sulfide solution $(\mathrm{v} / \mathrm{v})$, the sections were dehydrated with ascending concentrations of alcohol. CSAs of type I and type II fibers were measured using ImageJ. For small intestine, freshly dissected tissues were flushed with PBS and 4\% PFA, followed by fixation in $4 \%$ PFA overnight at $4{ }^{\circ} \mathrm{C}$, dehydrated, embedded in paraffin, and cut at $5 \mu \mathrm{m}$ thickness.

Grip strength, treadmill test, rotarod test and righting assay The forelimb grip strength of mice (4-month old) was assessed using a grip strength meter. Every mouse was tested for five times and the arithmetic average value of the records shall be as the measured value. For treadmill test, following familiarization test $(6 \mathrm{~m} / \mathrm{min}$ for $3 \mathrm{~min})$, the mouse (4-month old) was placed on a treadmill with a start speed of $10 \mathrm{~m} / \mathrm{min}$. Running speed was increased by $2 \mathrm{~m} / \mathrm{min}$ every $2 \mathrm{~min}$ until $30 \mathrm{~m} / \mathrm{min}$, and the distance was recorded when the mouse could no longer run. 
For rotarod performance test, the mouse (4-month old) was placed on a rotating rod at uniform motion (10 rpm) for $5 \mathrm{~min}$ and then an accelerating rotarod, which started with $4 \mathrm{rpm}$ and gradually increased to $40 \mathrm{rpm}$ in $5 \mathrm{~min}$. The mice were pre-trained for 2 days for adaptation. The duration time on the rotarod before the mice fell off was recorded. For righting assay, the mice on P5 were placed on its back to determine whether the mice could right themselves to normal posture with four paws on the ground.

MuSC isolation, expansion and in vitro differentiation

MuSCs were isolated according to the previously described methods. ${ }^{71,72,83}$ MuSCs were cultured on collagen-coated dish in $\mathrm{T}$ cell conditional medium for long-term expansion according to our reported protocol. ${ }^{72}$ Briefly, 10,000 MuSCs were seeded in a $3.5 \mathrm{~cm}$ dish and passaged every two days. The proliferation ability was determined during passaging period using cell counting and EdU labeling. MuSCs were differentiated in differentiation medium with $2 \%$ horse serum (Sigma). After being differentiated for $48 \mathrm{~h}$, the cells were stained with anti-MYH1 antibody (Merck Millipore, 05-716), and counterstained with DAPI for counting the number of nuclei in one fiber.

MuSC transplantation (in vivo differentiation)

MuSC transplantation was performed according to the described protocols ${ }^{84,85}$ with some modifications. Briefly, both hindlimbs of host $I L 2 r^{-1-}$ mice were exposed to $18 \mathrm{~Gy} Y$-radiations. Twenty-four hours before MuSC transplantation, $10 \mu \mathrm{M} \mathrm{CTX}(15 \mu \mathrm{L})$ is intramuscularly injected into mouse TA muscle via a $29 \mathrm{G}$ insulin syringe. In total $5 \times 10^{5}$ MuSCs were resuspended with $30 \mu \mathrm{L}$ of PBS and injected into TA muscle using $29 \mathrm{G}$ insulin syringe. TA muscles were collected in 4 weeks after cell transplantation and used for histological analysis.

\section{EdU labeling}

MuSCs were cultured in MuSC medium with EdU solution (Invitrogen; final concentration, $10 \mu \mathrm{M}$ ) for $2 \mathrm{~h}$ at $37^{\circ} \mathrm{C}$, followed by fixation with $3.7 \%$ PFA, permealized with $0.5 \%$ Triton X-100, reacted with Click-iT reaction cocktail and counterstained with Hoechst 33342.

\section{Skeletal preparation and staining}

Skeletal preparations were stained with Alcian blue and ARS. Briefly, the mice were executed, eviscerated and skinned. The samples were dehydrated with $95 \%$ ethanol for 3 days, and then were stained in Alcian blue solution for 3 days. After being fixed and cleared with $95 \%$ ethanol three times ( $1.5 \mathrm{~h}$ for each), the samples were treated with $2 \% \mathrm{KOH}$ for $3-4 \mathrm{~h}$ and stained with ARS solution for another 3-4 h. After staining, skeletons were cleared in $1 \% \mathrm{KOH} /$ $20 \%$ glycerol and stored in $100 \%$ glycerol up to several years.

\section{ELISA}

Insulin, PTH and TSH levels in blood were measured by ELISA kit (Ebioscience) according to the manufacturer's instructions. The whole blood was collected from retro-orbital bleeding.

EMG and ECG

EMG was performed under halothane anesthesia using 30-gauge concentric needle electrodes, with sampling of distal muscle group in left hindlimb. For ECG analysis, after removing the hair from chest using depilatory cream, the mouse was anesthetized and fixed on Vevo2100 (VisualSonics) by tighting the limbs on the mental detector to detect ECG. The ECG was recorded according to the manufacturer's instructions.

\section{Western blotting}

Dissected tissues were frozen with liquid nitrogen and stored in $-80^{\circ} \mathrm{C}$ refrigerators until protein extraction. Tissue homogenization was done by vortex in cell lysis buffer. After being separated on SDS-PAGE gels, the lysate was transferred to PVDF membranes and incubated with primary antibodies (anti-MBNL1, Abcam, ab108519; anti-SIX5, Abcam, ab113064; anti-DMWD, Santa Cruz, sc-167638; anti-DMPK, Santa Cruz, sc-13612) at $4{ }^{\circ} \mathrm{C}$ overnight, followed by incubation with secondary antibodies.

RNA-seq, gene expression and alternative splicing analyses MuSCs (Day 0) and differentiating cells (Day 1 and Day 2) were collected and subjected to RNA extraction using TRIZOL Reagent (Invitrogen). The total RNA was processed for RNA-seq by TruSeq RNA sample pre kit (Illumina) according to the manufacturer's instructions, and examined for a RIN number $(\geq 7)$ to inspect RNA integrity by an Agilent Bioanalyzer 2100 (Agilent Technologies). Qualified RNA was further purified by RNAClean XP Kit (Beckman Coulter, Inc.) and RNase-Free DNase Set (QIAGEN). Adaptors as well as low-quality base pairs were trimmed. RNA-seq was performed using Illumina Hiseq $X$ TEN. Then the preprocessed reads were aligned to the protein-coding genes of mouse reference genome (NCBI Mus musculus assembly GRCm38.p5) using hisat2 (version 2.0.5) and gene expression was quantified by stringtie. Alternative splicing events were detected by Astalavista (version 3.1) and profiled using MISO based on MISO Annotations version 2.0, which were performed by Shanghai Biotechnology Corporation. Moreover, the parameter settings of MISO referred to the reference. ${ }^{26}$ Venn diagram was plotted in RStudio.

RNA splicing and qRT-PCR

Total RNA was extracted from dissected tissues according to the manufacturer's instructions. $0.5 \mu \mathrm{g}$ of total RNA was reverse transcribed using a First Strand CDNA Synthesis kit (Toyobo). qPCR was carried out with THUNDERBIRD SYBR qPCR Mix (Toyobo) on a CFX connect Real-Time System (Bio-Rad). qPCR primers were listed in Supplementary information, Table S4. For alternative splicing analysis, the primers, which could separate the splice alternatives of target genes, ${ }^{86}$ were used to amplify the RNA variants, followed by electrophoresis on $2 \%$ agarose gel or SDSPAGE to calculate the splicing efficiency (percent of spliced in).

Small molecule screening

About 10,000 small compounds from Index Library of LifeArc (England) were screened on MuSCs planted in 384-well plates (3754) with differentiation medium. Before the compounds were added, the 384-well plate was coated with collagen and washed with PBS. 3500 MuSCs were plated by Multidrop and compounds (final concentration, $10 \mu \mathrm{M}$ ) were added by Mosquito. The MuSCs were differentiated in differentiation medium for two days and the images were captured using Operetta.

\section{Statistical analysis}

Quantitative values are presented as means \pm SEM, unless noted otherwise. Statistical differences between groups were determined by GraphPad Prism 6 using unpaired two-tailed $t$-test. No statistical method was used to predetermine sample size. Investigators were not blinded to outcome assessment.

Accession number

All RNA-seq data sets are available through GEO under the accession number: GSE103841.

\section{ACKNOWLEDGEMENTS}

$H S A^{L R}$ mice were obtained from Dr. Charles Thornton in the Wellstone Center at the University of Rochester. We thank Y. Chen and X. Ding from the Core Facility for Stem Cell Research at SIBCB for support with cell cultures and M. Chen from Core Facility for Chemical Biology for support with small-molecule screen. This study was supported by Genome Tagging Project, Fountain-Valley Life Sciences Fund of University of Chinese Academy of Sciences Education Foundation, and grants from the Ministry of Science and Technology of China (2019YFA0109900, 
2017YFA0102700), Chinese Academy of Sciences (XDB19010204, XDA16020400, QYZDJ-SSW-SMC023), the National Natural Science Foundation of China (31530048, $31821004,81672117,31900541,91649104,31671536)$, Shanghai Municipal Commission for Science and Technology (17411954900, 17JC1400900, 18ZR1446300 and 19ZR1423900), CAS-CSIRO Cooperative Research Program (GJHZ1504) and Innovation Promotion Association of the Chinese Academy of Sciences (to W.Y.).

\section{AUTHOR CONTRIBUTIONS}

J.L., P.H., and Q.Y. conceived the projects. Q.Y., Z.X., and C.Z. contributed to the haploid cell generation. Q.Y., Y.D., Q.L., Z.X. performed ICAHCI. Q.Y., H.W., N.L., L.J., Y.D., and W.Y. characterized the muscle phenotypes. Q.Y., Y.L., F.Z., C.D., and B.Z. analyzed heart phenotypes. Q.W. and L.B. performed rotarod experiments. Q.Y., Z.X., Q.Yu, Y.C., Y.M., and W.T. performed embryo transplantation. X.L., L.X., and W.C. performed EMG. K.W. analyzed RNA-seq data. J.L., P.H., and Q.Y. wrote and revised the manuscript with the help from all other authors.

\section{ADDITIONAL INFORMATION}

Supplementary information accompanies this paper at https://doi.org/10.1038/ s41422-019-0264-2.

Competing interests: The authors declare no competing interests.

\section{REFERENCES}

1. Korade-Mirnics, Z., Babitzke, P. \& Hoffman, E. Myotonic dystrophy: molecular windows on a complex etiology. Nucleic Acids Res. 26, 1363-1368 (1998).

2. Ranum, L. P. \& Day, J. W. Pathogenic RNA repeats: an expanding role in genetic disease. Trends Genet. 20, 506-512 (2004).

3. Udd, B. \& Krahe, R. The myotonic dystrophies: molecular, clinical, and therapeutic challenges. Lancet Neurol. 11, 891-905 (2012).

4. Brook, J. D. et al. Molecular basis of myotonic dystrophy: expansion of a trinucleotide (CTG) repeat at the $3^{\prime}$ end of a transcript encoding a protein kinase family member. Cell 68, 799-808 (1992).

5. Mahadevan, M. et al. Myotonic dystrophy mutation: an unstable CTG repeat in the 3' untranslated region of the gene. Science 255, 1253-1255 (1992).

6. $\mathrm{Fu}, \mathrm{Y}$. H. et al. An unstable triplet repeat in a gene related to myotonic muscular dystrophy. Science 255, 1256-1258 (1992).

7. Turner, C. \& Hilton-Jones, D. Myotonic dystrophy: diagnosis, management and new therapies. Curr. Opin. Neurol. 27, 599-606 (2014).

8. Pettersson, O. J., Aagaard, L., Jensen, T. G. \& Damgaard, C. K. Molecular mechanisms in DM1 - a focus on foci. Nucleic Acids Res. 43, 2433-2441 (2015).

9. Meola, G., Jones, K., Wei, C. \& Timchenko, L. T. Dysfunction of protein homeostasis in myotonic dystrophies. Histol. Histopathol. 28, 1089-1098 (2013).

10. Lee, J. E. \& Cooper, T. A. Pathogenic mechanisms of myotonic dystrophy. Biochem. Soc. Trans. 37, 1281-1286 (2009).

11. Davis, B. M., McCurrach, M. E., Taneja, K. L., Singer, R. H. \& Housman, D. E. Expansion of a CUG trinucleotide repeat in the $3^{\prime}$ untranslated region of myotonic dystrophy protein kinase transcripts results in nuclear retention of transcripts. Proc. Natl Acad. Sci. USA 94, 7388-7393 (1997).

12. Maeda, M. et al. Identification, tissue-specific expression, and subcellular localization of the 80 - and $71-\mathrm{kDa}$ forms of myotonic dystrophy kinase protein. J. Biol. Chem. 270, 20246-20249 (1995).

13. Fu, Y. H. et al. Decreased expression of myotonin-protein kinase messenger RNA and protein in adult form of myotonic dystrophy. Science 260, 235-238 (1993).

14. Otten, A. D. \& Tapscott, S. J. Triplet repeat expansion in myotonic dystrophy alters the adjacent chromatin structure. Proc. Natl Acad. Sci. USA 92, 5465-5469 (1995).

15. Iyer, R. R., Pluciennik, A., Napierala, M. \& Wells, R. D. DNA triplet repeat expansion and mismatch repair. Annu. Rev. Biochem. 84, 199-226 (2015).

16. Dion, V. \& Wilson, J. H. Instability and chromatin structure of expanded trinucleotide repeats. Trends Genet. 25, 288-297 (2009).

17. Usdin, K. The biological effects of simple tandem repeats: lessons from the repeat expansion diseases. Genome Res. 18, 1011-1019 (2008).

18. Klesert, T. R., Otten, A. D., Bird, T. D. \& Tapscott, S. J. Trinucleotide repeat expansion at the myotonic dystrophy locus reduces expression of DMAHP. Nat. Genet. 16, 402-406 (1997).

19. Thornton, C. A., Wymer, J. P., Simmons, Z., McClain, C. \& Moxley, R. T. 3rd Expansion of the myotonic dystrophy CTG repeat reduces expression of the flanking DMAHP gene. Nat. Genet. 16, 407-409 (1997).

20. Gennarelli, M. et al. Reduction of the DM-associated homeo domain protein (DMAHP) mRNA in different brain areas of myotonic dystrophy patients. Neuromuscul. Disord. 9, 215-219 (1999).
21. Inukai, A. et al. Reduced expression of DMAHP/SIX5 gene in myotonic dystrophy muscle. Muscle Nerve 23, 1421-1426 (2000).

22. Yanovsky-Dagan, S. et al. Uncovering the role of hypermethylation by CTG expansion in myotonic dystrophy type 1 using mutant human embryonic stem cells. Stem Cell Rep. 5, 221-231 (2015).

23. Alwazzan, M., Newman, E., Hamshere, M. G. \& Brook, J. D. Myotonic dystrophy is associated with a reduced level of RNA from the DMWD allele adjacent to the expanded repeat. Hum. Mol. Genet. 8, 1491-1497 (1999).

24. Frisch, R. et al. Effect of triplet repeat expansion on chromatin structure and expression of DMPK and neighboring genes, SIX5 and DMWD, in myotonic dystrophy. Mol. Genet Metab. 74, 281-291 (2001).

25. Hamshere, M. G., Newman, E. E., Alwazzan, M., Athwal, B. S. \& Brook, J. D. Transcriptional abnormality in myotonic dystrophy affects DMPK but not neighboring genes. Proc. Natl Acad. Sci. USA 94, 7394-7399 (1997).

26. Thomas, J. D. et al. Disrupted prenatal RNA processing and myogenesis in congenital myotonic dystrophy. Genes Dev. 31, 1122-1133 (2017).

27. Timchenko, L. T., Timchenko, N. A., Caskey, C. T. \& Roberts, R. Novel proteins with binding specificity for DNA CTG repeats and RNA CUG repeats: implications for myotonic dystrophy. Hum. Mol. Genet. 5, 115-121 (1996).

28. Philips, A. V., Timchenko, L. T. \& Cooper, T. A. Disruption of splicing regulated by a CUG-binding protein in myotonic dystrophy. Science 280, 737-741 (1998).

29. Fardaei, M. et al. Three proteins, MBNL, MBLL and MBXL, co-localize in vivo with nuclear foci of expanded-repeat transcripts in DM1 and DM2 cells. Hum. Mol. Genet. 11, 805-814 (2002).

30. Miller, J. W. et al. Recruitment of human muscleblind proteins to (CUG)(n) expansions associated with myotonic dystrophy. EMBO J. 19, 4439-4448 (2000).

31. Mankodi, A. et al. Muscleblind localizes to nuclear foci of aberrant RNA in myotonic dystrophy types 1 and 2. Hum. Mol. Genet .10, 2165-2170 (2001).

32. Fardaei, M., Larkin, K., Brook, J. D. \& Hamshere, M. G. In vivo co-localisation of MBNL protein with DMPK expanded-repeat transcripts. Nucleic Acids Res. 29, 2766-2771 (2001)

33. Reddy, S. et al. Mice lacking the myotonic dystrophy protein kinase develop a late onset progressive myopathy. Nat. Genet. 13, 325-335 (1996).

34. Jansen, G. et al. Abnormal myotonic dystrophy protein kinase levels produce only mild myopathy in mice. Nat. Genet. 13, 316-324 (1996).

35. Sarkar, P. S. et al. Heterozygous loss of Six 5 in mice is sufficient to cause ocular cataracts. Nat. Genet. 25, 110-114 (2000).

36. Klesert, T. R. et al. Mice deficient in Six5 develop cataracts: implications for myotonic dystrophy. Nat. Genet. 25, 105-109 (2000).

37. Kanadia, R. N. et al. A muscleblind knockout model for myotonic dystrophy. Science 302, 1978-1980 (2003).

38. Gomes-Pereira, M., Cooper, T. A. \& Gourdon, G. Myotonic dystrophy mouse models: towards rational therapy development. Trends Mol. Med. 17, 506-517 (2011).

39. Mankodi, A. et al. Myotonic dystrophy in transgenic mice expressing an expanded CUG repeat. Science 289, 1769-1773 (2000).

40. van den Broek, W. J. et al. Somatic expansion behaviour of the (CTG)n repeat in myotonic dystrophy knock-in mice is differentially affected by Msh3 and Msh6 mismatch-repair proteins. Hum. Mol. Genet. 11, 191-198 (2002).

41. Larkin, K. \& Fardaei, M. Myotonic dystrophy-a multigene disorder. Brain Res. Bull. 56, 389-395 (2001).

42. Groenen, P. \& Wieringa, B. Expanding complexity in myotonic dystrophy. Bioessays 20, 901-912 (1998).

43. Zhong, C. et al. CRISPR-Cas9-mediated genetic screening in mice with haploid embryonic stem cells carrying a guide RNA library. Cell Stem Cell 17, 221-232 (2015).

44. Yang, H. et al. Generation of genetically modified mice by oocyte injection of androgenetic haploid embryonic stem cells. Cell 149, 605-617 (2012).

45. Li, W. et al. Androgenetic haploid embryonic stem cells produce live transgenic mice. Nature 490, 407-411 (2012).

46. Hermans, M. C. et al. Structural and functional cardiac changes in myotonic dystrophy type 1: a cardiovascular magnetic resonance study. J. Cardiovasc. Magn. Reson. 14, 48 (2012).

47. Westerlaken, J. H., Van der Zee, C. E., Peters, W. \& Wieringa, B. The DMWD protein from the myotonic dystrophy (DM1) gene region is developmentally regulated and is present most prominently in synapse-dense brain areas. Brain Res. 971, 116-127 (2003).

48. Eriksson, M. et al. Independent regulation of the myotonic dystrophy 1 locus genes postnatally and during adult skeletal muscle regeneration. J. Biol. Chem. 275, 19964-19969 (2000).

49. Jansen, G. et al. Structural organization and developmental expression pattern of the mouse WD-repeat gene DMR-N9 immediately upstream of the myotonic dystrophy locus. Hum. Mol. Genet. 4, 843-852 (1995).

50. Jansen, G. et al. Characterization of the myotonic dystrophy region predicts multiple protein isoform-encoding mRNAs. Nat. Genet. 1, 261-266 (1992). 
51. Boucher, C. A. et al. A novel homeodomain-encoding gene is associated with a large CpG island interrupted by the myotonic dystrophy unstable (CTG)n repeat. Hum. Mol. Genet. 4, 1919-1925 (1995).

52. Sarkar, P. S., Paul, S., Han, J. \& Reddy, S. Six5 is required for spermatogenic cell survival and spermiogenesis. Hum. Mol. Genet. 13, 1421-1431 (2004).

53. $\mathrm{Wu}, \mathrm{Y}$. et al. Correction of a genetic disease in mouse via use of CRISPR-Cas9. Cell Stem Cell 13, 659-662 (2013).

54. Ho, G., Cardamone, M. \& Farrar, M. Congenital and childhood myotonic dystrophy: current aspects of disease and future directions. World J. Clin. Pediatr. 4, 66-80 (2015).

55. Panaite, P. A., Kuntzer, T., Gourdon, G., Lobrinus, J. A. \& Barakat-Walter, I. Functional and histopathological identification of the respiratory failure in a DMSXL transgenic mouse model of myotonic dystrophy. Dis. Model Mech. 6, 622-631 (2013).

56. Miyagi, N., lisaka, H., Yasuda, K. \& Kaneda, K. Onset of ossification of the tarsal bones in congenital clubfoot. J. Pediatr. Orthop. 17, 36-40 (1997).

57. Mateos-Aierdi, A. J. et al. Muscle wasting in myotonic dystrophies: a model of premature aging. Front Aging Neurosci. 7, 125 (2015).

58. Groh, W. J. et al. Electrocardiographic abnormalities and sudden death in myotonic dystrophy type 1. N. Engl. J. Med. 358, 2688-2697 (2008).

59. Ward, A. J., Rimer, M., Killian, J. M., Dowling, J. J. \& Cooper, T. A. CUGBP1 overexpression in mouse skeletal muscle reproduces features of myotonic dystrophy type 1. Hum. Mol. Genet. 19, 3614-3622 (2010).

60. Tohgi, H., Kawamorita, A., Utsugisawa, K., Yamagata, M. \& Sano, M. Muscle histopathology in myotonic dystrophy in relation to age and muscular weakness. Muscle Nerve 17, 1037-1043 (1994).

61. Dahlqvist, J. R., Orngreen, M. C., Witting, N. \& Vissing, J. Endocrine function over time in patients with myotonic dystrophy type 1. Eur. J. Neurol. 22, 116-122 (2015).

62. Botta, A. et al. The CTG repeat expansion size correlates with the splicing defects observed in muscles from myotonic dystrophy type 1 patients. J. Med. Genet. 45, 639-646 (2008)

63. Vihola, A. et al. Differences in aberrant expression and splicing of sarcomeric proteins in the myotonic dystrophies DM1 and DM2. Acta Neuropathol. 119, 465-479 (2010).

64. Lee, K. Y. et al. Compound loss of muscleblind-like function in myotonic dys trophy. EMBO Mol. Med. 5, 1887-1900 (2013).

65. Furling, D. et al. Defective satellite cells in congenital myotonic dystrophy. Hum. Mol. Genet. 10, 2079-2087 (2001).

66. Beffy, P. et al. Altered signal transduction pathways and induction of autophagy in human myotonic dystrophy type 1 myoblasts. Int J. Biochem. Cell Biol. 42 1973-1983 (2010).

67. Thornell, L. E. et al. Satellite cell dysfunction contributes to the progressive muscle atrophy in myotonic dystrophy type 1. Neuropathol. Appl. Neurobiol. 35, 603-613 (2009)

68. Bigot, A. et al. Large CTG repeats trigger p16-dependent premature senescence in myotonic dystrophy type 1 muscle precursor cells. Am. J. Pathol. 174, 1435-1442 (2009).

69. Beaulieu, D. et al. Abnormal prostaglandin E2 production blocks myogenic differentiation in myotonic dystrophy. Neurobiol. Dis. 45, 122-129 (2012).

70. Amack, J. D., Reagan, S. R. \& Mahadevan, M. S. Mutant DMPK 3'-UTR transcripts disrupt C2C12 myogenic differentiation by compromising MyoD. J. Cell Biol. 159, 419-429 (2002).
71. Sacco, A., Doyonnas, R., Kraft, P., Vitorovic, S. \& Blau, H. M. Self-renewal and expansion of single transplanted muscle stem cells. Nature 456, 502-506 (2008).

72. Fu, X. et al. Combination of inflammation-related cytokines promotes long-term muscle stem cell expansion. Cell Res. 25, 655-673 (2015).

73. Bentzinger, C. F., Wang, Y. X. \& Rudnicki, M. A. Building muscle: molecular regulation of myogenesis. Cold Spring Harb. Perspect. Biol. 4, a008342 (2012).

74. Ma, Z. et al. PTC-bearing mRNA elicits a genetic compensation response via Upf3a and COMPASS components. Nature 568, 259-263 (2019).

75. Li, Q. et al. Temporal regulation of prenatal embryonic development by paternal imprinted loci. Sci China Life Sci. https://doi.org/10.1007/s11427-019-9817-6 (2019).

76. Hao, M. et al. Muscleblind-like 2 (Mbnl2)-deficient mice as a model for myotonic dystrophy. Dev. Dyn. 237, 403-410 (2008).

77. Randolph, M. E. \& Pavlath, G. K. A muscle stem cell for every muscle: variability of satellite cell biology among different muscle groups. Front Aging Neurosci. 7, 190 (2015).

78. Gifford, C. A. et al. Oligogenic inheritance of a human heart disease involving a genetic modifier. Science 364, 865-870 (2019).

79. $\mathrm{Wu}, \mathrm{Y}$. et al. Correction of a genetic disease by CRISPR-Cas9-mediated gene editing in mouse spermatogonial stem cells. Cell Res. 25, 67-79 (2015).

80. Zhong, C. \& Li, J. Efficient generation of gene-modified mice by haploid embryonic stem cell-mediated semi-cloned technology. Methods Mol. Biol. 1498 121-133 (2017).

81. Crist, C. G., Montarras, D. \& Buckingham, M. Muscle satellite cells are primed for myogenesis but maintain quiescence with sequestration of Myf5 mRNA targeted by microRNA-31 in mRNP granules. Cell Stem Cell 11, 118-126 (2012).

82. Pestronk, G. J., Kaiser, K. K. \& Brooke, M. H. ATPase stain in muscle histochemistry. Muscle Nerve 15, 258 (1992).

83. Gilbert, P. M. et al. Substrate elasticity regulates skeletal muscle stem cell selfrenewal in culture. Science 329, 1078-1081 (2010).

84. Montarras, D. et al. Direct isolation of satellite cells for skeletal muscle regeneration. Science 309, 2064-2067 (2005).

85. Motohashi, N., Asakura, Y. \& Asakura, A. Isolation, culture, and transplantation of muscle satellite cells. J. Vis. Exp. 86, 50846 (2014).

86. Lin, X. et al. Failure of MBNL1-dependent post-natal splicing transitions in myotonic dystrophy. Hum. Mol. Genet. 15, 2087-2097 (2006).

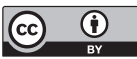

Open Access This article is licensed under a Creative Commons Attribution 4.0 International License, which permits use, sharing adaptation, distribution and reproduction in any medium or format, as long as you give appropriate credit to the original author(s) and the source, provide a link to the Creative Commons license, and indicate if changes were made. The images or other third party material in this article are included in the article's Creative Commons license, unless indicated otherwise in a credit line to the material. If material is not included in the article's Creative Commons license and your intended use is not permitted by statutory regulation or exceeds the permitted use, you will need to obtain permission directly from the copyright holder. To view a copy of this license, visit http://creativecommons. org/licenses/by/4.0/.

(c) The Author(s) 2019 